\title{
Domain Regeneration for Cross-Database Micro-Expression Recognition
}

\author{
Yuan Zong, Wenming Zheng*, Member, IEEE, Xiaohua Huang, Jingang Shi, Zhen Cui, Member, IEEE, \\ and Guoying Zhao, Senior Member, IEEE
}

\begin{abstract}
Recently, micro-expression recognition has attracted lots of researchers' attention due to its potential value in many practical applications, e.g., lie detection. In this paper, we investigate an interesting and challenging problem in micro-expression recognition, i.e., cross-database micro-expression recognition, in which the training and testing samples come from different micro-expression databases. Under this problem setting, the consistent feature distribution between the training and testing samples originally existing in conventional micro-expression recognition would be seriously broken and hence the performance of most current well-performing micro-expression recognition methods may sharply drop. In order to overcome it, we propose a simple yet effective framework called Domain Regeneration (DR) in this paper. DR framework aims at learning a domain regenerator to regenerate the micro-expression samples from source and target databases respectively such that they can abide by the same or similar feature distributions. Thus, we are able to use the classifier learned based on the labeled source micro-expression samples to predict the label information of the unlabeled target micro-expression samples. To evaluate the proposed DR framework, we conduct extensive cross-database micro-expression recognition experiments designed based on SMIC and CASME II databases. Experimental results show that compared with recent state-of-the-art cross-database emotion recognition methods, the proposed DR framework has more promising performance.
\end{abstract}

Index Terms-Cross-database micro-expression recognition, micro-expression recognition, domain adaptation, transfer learning.

Manuscript received July 30, 2017; revised December 21, 2017; accepted January 14, 2018. This work was supported by the National Basic Research Program of China under Grant 2015CB351704, the National Natural Science Foundation of China under Grant 61231002, Grant 61572009, Grant 61772276, and Grant 61602244, China Scholarship Council, the Scientific Research Foundation of Graduate School of Southeast University under Grant YBJJ1774, Academy of Finland, Tekes Fidipro Program, the Strategic Funds of the University of Oulu, Finland, Infotech Oulu, and Jorma Ollila Grant of Nokia Foundation.

Y. Zong is with the Key Laboratory of Child Development and Learning Science of Ministry of Education, School of Biological Sciences and Medical Engineering, Southeast University, Nanjing 210096, China, and also with the Center for Machine Vision and Signal Analysis, Faulty of Information Technology and Electrical Engineering, University of Oulu, Oulu FI-90014, Finland (e-mail: xhzongyuan@ seu.edu.cn).

W. Zheng is with the Key Laboratory of Child Development and Learning Science of Ministry of Education, School of Biological Sciences and Medical Engineering, Southeast University, Nanjing 210096, China (e-mail: wenming_zheng@seu.edu.cn).

X. Huang, J. Shi and G. Zhao are with the Center for Machine Vision and Signal Analysis, Faulty of Information Technology and Electrical Engineering, University of Oulu, Oulu FI-90014, Finland (e-mail: \{xiaohua.huang, jingang.shi, guoying.zhao $\} @$ oulu.fi).

Z. Cui is with the School of Computer Science and Engineering, Nanjing University of Science and Technology, Nanjing 210094, China (email: zhen.cui@njust.edu.cn).

The asterisk indicates corresponding author

\section{INTRODUCTION}

A $\mathrm{S}$ one type of particular dynamic facial expressions, micro-expressions can reveal human beings' hidden emotions which they try to conceal [1]. Thus, automatically recognizing micro-expressions by a machine will have great values in many practical application fields such as clinical diagnosis [2], interrogation [3], and security [4]. However, compared with ordinary dynamic facial expressions, micro-expressions have much lower intensity and shorter duration, which makes automatic micro-expression recognition be a very difficult task. Nevertheless, micro-expression recognition still becomes one of recent attractive research topics and in recent years extensive effective methods have been proposed to deal with this challenging task [5], [6]. But it can be noted that most of current micro-expression recognition methods are developed and evaluated in the scenario, where the training and testing samples belong to the same micro-expression database. In this case, it can be thought that such training and testing samples abide by the same or similar feature distributions. In the practical applications, however, the training and testing microexpression samples may come from two different databases. Clearly, they are recorded under different environments or by different equipment. Hence in this scenario, the training and testing micro-expression samples would be quite different, which causes that their feature distributions would have wide difference. Because of this, the performance of most current micro-expression recognition methods may decrease greatly. In order to solve this problem and develop more practical micro-expression recognition methods, it is very worthwhile to investigate cross-database micro-expression recognition problem, where the training and testing samples belong to two different micro-expression databases. For convenience, we will refer the training database as the source domain (database) and the testing database as the target domain (database) in crossdatabase micro-expression recognition throughout this paper.

In the work of [7], Yan et al. roughly divided cross-database facial expression recognition problem into two cases including semi-supervised case and unsupervised case. The major difference between these two cases is whether we have access to the label information of target domain. Similarly, cross-database micro-expression recognition problem can follow this categorization. In this paper, we will focus on the unsupervised cross-database micro-expression recognition, in which the label information of the source micro-expression database is available, while the samples from the target micro-expression database are all unlabeled. To deal with this problem, we have 
proposed a simple yet effective method called Target Sample Re-Generator (TSRG) in our conference work of [8]. TSRG aims at learning a sample regenerator for source and target micro-expression samples. By using the learned sample regenerator, the regenerated source micro-expression samples are still the original source ones, while the regenerated target micro-expression samples become a new set of samples which are different from their original forms but have the same or similar feature distributions with the source micro-expression samples. Subsequently, we are able to learn a classifier such as support vector machine (SVM) based on the labeled source micro-expression samples and then use it to predict the labels of the unlabeled regenerated target micro-expression samples.

In this paper, we will re-investigate the unsupervised crossdatabase micro-expression recognition problem and extend our conference work of TSRG [8] to a generalized framework called Domain Regeneration. DR framework inherits the basic idea of TSRG that is pursuing learning a sample regenerator to regenerate the source and target micro-expression to eliminate their feature distribution mismatch. To differentiate with TSRG, we name the sample rengerator under the DR framework domain regenerator throughout the paper. In summary, besides the contribution of TSRG in our preliminary work [8], this paper contains following three main contributions:

1) We propose a DR framework for dealing with unsupervised cross-database micro-expression recognition. The TSRG proposed in our conference work of [8] can be included to this framework and be interpreted as a DR based domain regenerator, i.e., DR in the original Feature Space with unchanged Source domain (DRFSS).

2) Besides DRFS-S (TSRG), we propose two more novel domain regenerators under the DR framework to deal with unsupervised cross-database micro-expression recognition problem. One is DR in the original Feature Space with unchanged Target domain (DRFS-T). The other is DR in the Label Space (DRLS), which fully leverages the label information provided by the source domain.

3) Compared with our preliminary work of TSRG [8], we conduct more extensive cross-database micro-expression recognition experiments to evaluate the proposed DR framework based methods including DRFS-S (TSRG), DRFS-T and DRLS.

The rest of this paper is organized as follows: Section II reviews recent works about micro-expression recognition and cross-database emotion (including facial expression and speech emotion) recognition which are closely related to crossdatabase micro-expression recognition topic. In Section III, we introduce our proposed DR framework for cross-database micro-expression recognition in detail. For evaluating DR framework, extensive cross-database micro-expression recognition experiments on SMIC and CASME II databases are conducted in Section IV. Finally, the conclusion of this paper is drawn in Section V.

\section{RELATED WORKS}

\section{A. Micro-Expression Recognition}

The micro-expression recognition research can be early traced to the work of [1], in which Pfister et al. proposed to use local binary pattern from three orthogonal planes (LBP-TOP) [9] to describe micro-expression video clips for micro-expression recognition task. Their experimental results show that LBP-TOP is effective for micro-expression recognition problem. Following Pfister et al.'s work, RuizHernandez et al. [10] employed re-parameterization of second order Gaussian jet to boost LBP-TOP such that LBP-TOP is more applicable to micro-expression recognition. For better describing micro-expressions, Wang et al. [11] proposed a novel spatio-temporal descriptor called LBP with six intersection points (LBP-SIP) which can reduce the redundant information in LBP-TOP. Subsequently, lots of spatio-temporal descriptors are developed for micro-expression recognition tasks, such as spatio-temporal LBP with integration projection (STLBP-IP) [12], completed local quantized patternTOP (CLQP-TOP) [13], histogram of oriented gradient-TOP (HOG-TOP) [5], and histogram of image gradient orientationTOP (HIGO-TOP) [5]. Furthermore, different from the above spatio-temporal descriptors, other types of micro-expression features are also investigated by researchers. Among them, it is worth mentioning the works of [14], [15], in which Liu et al. and $\mathrm{Xu}$ et al. respectively designed novel features, i.e., main directional mean optical (MDMO) and facial dynamics map (FDM), to describe micro-expressions.

On the other hand, in recent years, some researchers investigated micro-expression recognition from a new angle. They aim at leveraging other important information, which contributes to distinguishing micro-expressions, from microexpression video clips to boost the performance of the spatiotemporal descriptors. For example, in the work of [16], Wang et al. proposed to use robust principal component analysis (RPCA) [17] to extract the background information from the micro-expression video clips and then extract the spatiotemporal descriptor from the background information to describe the micro-expressions. Moreover, Wang et al. [18], [19] also proposed a color space decomposition method called tensor independent color space (TICS) to utilize the color information for micro-expression recognition. Besides, according to the facial action coding system (FACS) [20], Wang et al. [18], [19] and Liu et al. [14] designed two groups of regions of interest (ROIs), which contributes to distinguishing microexpressions, for micro-expression feature extraction.

More recently, deep learning methods have also been applied to micro-expression recognition. For example, Kim et al. [21] proposed a deep learning framework consisting of popular convolutional neural network (CNN) [22] and long short-term memory (LSTM) recurrent network [23] for microexpression recognition. In this framework, the representative expression-states frames of each micro-expression video clip are first selected to train a CNN. Then, the CNN feature of each image frame in a video clip is extracted to train a LSTM network for recognizing micro-expressions. 


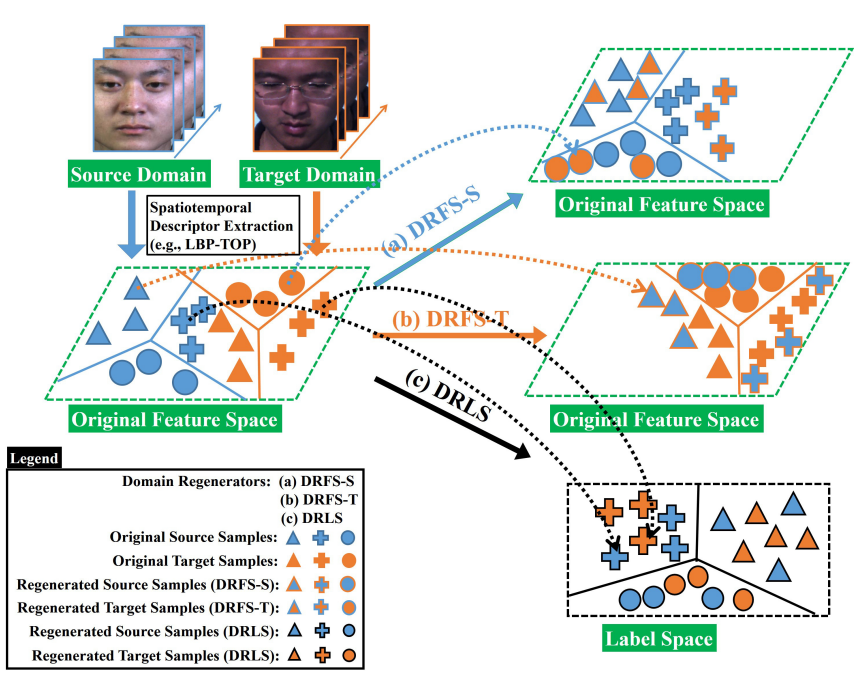

Fig. 1. Domain Regeneration (DR) framework for dealing with crossdatabase micro-expression recognition. Under this framework, we design three domain regenerators including (a) DRFS-S: Domain Regeneration in the original Feature Space with unchanged Source domain, (b) DRFS-T: Domain Regeneration in the original Feature Space with unchanged Target domain, and (c) DRLS: Domain Regeneration in the Label Space, for cross-database micro-expression recognition.

\section{B. Cross-Database Emotion Recognition}

Since cross-database micro-expression recognition problem has not yet been investigated, in this section we review recent works about other modality based cross-database emotion recognition that is closely related to cross-database microexpression recognition including cross-database facial expression recognition and cross-database speech emotion recognition. In recent years, these two challenging and interesting problems have gained lots of researchers' attention.

For cross-database facial expression recognition and its related problems, various effective methods [24], [25], [26], [7], [27], [28] have been proposed. For example, in the works of [24], [25], Chu et al. proposed a novel method called selective transfer machine (STM) for personalized (cross-subject) facial action units detection problem. STM is able to utilize the target samples to learn a set of weights for the source samples such that the weighted source samples would have the same or similar feature distributions with the target samples. Consequently, the classifier trained based on the weighted source samples could also be suitable for distinguishing the target samples. Recently, Sangineto et al. [26] investigated the cross-domain facial expression recognition problem by using a transductive parameter transfer method. They proposed a novel classifier parameter transfer method to directly transfer knowledge about the parameters of source person-specific classifiers to the target individuals such that the target classifier can accurately predict the expressions of target samples. More recently, Yan et al. [7] proposed an unsupervised domainadaptive dictionary learning (UDADL) model to cope with the unsupervised cross-database facial expression recognition problem and achieved a promising result. In addition, Zheng et al. [27], [28] proposed a transductive transfer subspace learning framework to deal with cross-pose and cross-database cases in facial expression recognition. In this framework, an auxiliary set is selected from the unlabeled target samples for learning a subspace together with the labeled source samples. In such a subspace, the source and target samples would be enforced to abide by the same feature distribution and hence the classifier trained by the source samples can predict the expressions of the target samples.

The earliest cross-database speech emotion recognition research may be the work of [29], in which Schuller et al. proposed to employ a series of normalization methods to investigate cross-database speech emotion recognition problem and conducted extensive cross-database experiments on many speech emotion databases. From then on, a variety of interesting methods have been proposed to deal with this challenging problem [30], [31], [32], [33], [34], [35], [36], [37]. For example, Hassan et al. [30] proposed an importanceweighted support vector machine (IW-SVM) to handle crossdatabase speech emotion recognition. In this method, they first used three transfer learning [38] methods, i.e., kernel mean matching (KMM) [39], Kullback-Leibler importance estimation procedure (KLIEP) [40], and unconstrained leastsquares importance fitting (uLSIF) [41] to learn the importance weights for target samples with respect to source samples and then incorporated the learned weights into SVM to obtain the IW-SVM. In the works of [31], [32], [33], Deng et al. proposed a series of auto-encoder based domain adaptation methods by leveraging various auto-encoder based networks to learn a common representation between the source and target samples for cross-database speech emotion recognition problem. Recently, Song et al. [35] proposed a transfer nonnegative matrix factorization method for coping with crossdatabase speech emotion recognition, in which the maximum mean discrepancy (MMD) [42] is introduced to eliminate the feature distribution difference between source and target speech databases.

From the above cross-database emotion recognition methods, it is clear that the basic idea of these methods can be almost categorized into two types. The first type of methods target at learning the importance weights for source or target samples to balance the feature distribution difference between source and target databases, while the second type of methods achieve this goal by learning a common subspace. Different from the above types, our DR framework is developed to this end from a new angle, i.e., learning a domain regenerator to regenerate source and target domains such that their corresponding samples can abide by the same and similar feature distributions.

\section{DOMAIN REgEnERATION FOR CROSS-DATABASE MICRO-EXPRESSION RECOGNITION}

\section{A. Basic Idea of Domain Regeneration}

In this section, we address our Domain Regeneration (DR) framework in detail. For better understanding Domain Regeneration (DR) framework, we draw a picture shown in Fig. 1 to illustrate the basic idea of DR framework and show how it works for dealing with cross-database microexpression recognition problem. As depicted in Fig. 1, it can be seen that the goal of the proposed DR framework 
is to learn a domain regenerator, which can regenerate the micro-expression samples from source and target domains in a predefined domain (subspace), e.g., the original feature space. By using the domain regenerators, the regenerated source and target micro-expression samples would become abiding by the same or similar feature distributions. After that, we are able to learn a classifier, e.g., SVM, based on the microexpression label information provided by source domain and the regenerated source samples, and then use this classifier to predict the micro-expression categories of the regenerated target samples.

As Fig. 1 shows, in this paper, we design three kinds of domain regenerators including (a) Domain Regeneration in the original Feature space with unchanged Source domain (DRFS-S), (b) Domain Regeneration in the original Feature space with unchanged Target domain (DRFS-T), and (c) Domain Regeneration in the Label space (DRLS), under the proposed DR framework. It is also clear to see that the major difference among three domain regenerators is the predefined subspace for performing domain regeneration. Specifically, DRLS performs domain regeneration in the label space which is spanned according to the label information provided by source domain. Instead, by using the DRFS-S and DRFS-T, the micro-expression samples from source and target domains are regenerated still in the original feature space where the source and target samples originally lie.

\section{B. Domain Regeneration Framework}

Suppose we have feature matrices $\mathbf{X}_{s} \in \mathbb{R}^{d \times n_{s}}$ and $\mathbf{X}_{t} \in$ $\mathbb{R}^{d \times n_{t}}$ of source and target micro-expression samples from two different databases, where $d$ is the dimension of feature vector and $n_{s}$ and $n_{t}$ denote the numbers of source samples and target samples, respectively. According to the problem setting of unsupervised cross-database micro-expression recognition, the label information of source domain is available for us and herein we use a label matrix $\mathbf{L}_{s}=\left[\mathbf{l}_{1}, \cdots, \mathbf{l}_{n_{s}}\right] \in \mathbb{R}^{c \times n_{s}}$ to denote the label information of the source domain, where $c$ is the number of micro-expression categories. The $i^{\text {th }}$ column $\mathbf{l}_{i}=\left[l_{i, 1}, \cdots, l_{i, c}\right]^{T}$ of $\mathbf{L}_{s}$ is a column vector whose elements take a binary value 0 or 1 according to the following rule:

$l_{i, j}= \begin{cases}1, & \text { if } \mathbf{x}_{s}^{i} \text { belongs to the } j^{t h} \text { micro-expression class; } \\ 0, & \text { otherwise }\end{cases}$

where $\mathbf{x}_{s}^{i}$ is the $i^{\text {th }}$ column of $\mathbf{X}_{s}$. Note that the above microexpression features can be any spatiotemporal descriptor for describing micro-expressions such as LBP-TOP [9], [1], LBPSIP [11], and MDMO [14].

To achieve the goal depicted in Fig. 1, we should first design a well-defined subspace for DR framework to serve as the domain regeneration platform, which means that the regenerated source and target micro-expression samples will lie in this subspace after regeneration. Let us define this satisfactory subspace a $r$-dimensional one and denote the desired regenerated source and target samples in this subspace by $\mathbf{R}_{s} \in \mathbb{R}^{r \times n_{s}}$ and $\mathbf{R}_{t} \in \mathbb{R}^{r \times n_{t}}$. Then, we are able to formulate the optimization problem to learn a domain regenerator $G$ under the DR framework, which can transform the source and target micro-expression samples from the original feature space to the predefined subspace, as follows:

$$
\min _{G}\left\|\mathbf{R}_{\mathcal{D}}-G\left(\mathbf{X}_{\mathcal{D}}\right)\right\|_{F}^{2}, \quad(\mathcal{D} \in\{s, t\}) .
$$

Secondly, to ensure the regenerated source and target samples in the predefined subspace will have same or similar feature distributions, which is shown in Fig. 1, we should also design one more term for the objective function of the proposed DR framework. Let $f_{G}\left(\mathbf{X}_{s}, \mathbf{X}_{t}\right)$ be such a satisfactory term and its detail will be introduced in what follows. Then, by using $f_{G}\left(\mathbf{X}_{s}, \mathbf{X}_{t}\right)$ as the regularization term, we can obtain the optimization problem of DR framework as follows:

$$
\min _{G}\left\|\mathbf{R}_{\mathcal{D}}-G\left(\mathbf{X}_{\mathcal{D}}\right)\right\|_{F}^{2}+\lambda f_{G}\left(\mathbf{X}_{s}, \mathbf{X}_{t}\right), \quad(\mathcal{D} \in\{s, t\}),
$$

where $\lambda$ is the trade-off parameter to control the balance between these two terms in DR objective function.

It is notable that with the source or target samples $\mathbf{X}_{\mathcal{D}}$ as input, the output $G\left(\mathbf{X}_{\mathcal{D}}\right)$ of the domain regenerator $G$ are hoped to be the desired regenerated source or target samples $\mathbf{R}_{\mathcal{D}}$. This goal can actually be easy to achieve by using a combination of the kernel mapping operation and the linear projection operation, which are two typical operations in subspace learning. More specifically, a suitable domain regenerator $G$ can first map the source or target samples from the original feature space into a reproduced kernel Hilbert space (RKHS) by a kernel mapping operator $\phi$ and subsequently transform the infinite-dimensional source or target features in RKHS back to the predefined subspace by a projection matrix $\phi(\mathbf{C}) \in \mathbb{R}^{\infty \times r}$. Following this idea, the domain regenerator $G$ can be finally defined as $G(\cdot)=\phi(\mathbf{C})^{T} \phi(\cdot)$. Then the optimization problem of DR framework in Eq. (2) can be rewritten as:

$$
\min _{\phi(\mathbf{C})}\left\|\mathbf{R}_{\mathcal{D}}-\phi(\mathbf{C})^{T} \phi\left(\mathbf{X}_{\mathcal{D}}\right)\right\|_{F}^{2}+\lambda f_{G}\left(\mathbf{X}_{s}, \mathbf{X}_{t}\right) .
$$

Besides obtaining expected regenerated source or target samples $\mathbf{R}_{\mathcal{D}}$, the domain regenerator $G$ consisting of kernel mapping and linear projection operators can also bring an important benefit to construct $f_{G}\left(\mathbf{X}_{s}, \mathbf{X}_{t}\right)$ for the proposed DR framework. It is known that we are able to eliminate the feature distribution difference between two different feature sets by minimizing their maximum mean discrepancy (MMD) [42] which is defined in a RKHS. Regarding $f_{G}\left(\mathbf{X}_{s}, \mathbf{X}_{t}\right)$ in our DR framework, we can formulate it as the MMD between source and target samples in the RKHS produced by $\phi$, which is expressed as:

$$
\operatorname{MMD}\left(\mathbf{X}_{s}, \mathbf{X}_{t}\right)=\left\|\frac{1}{n_{s}} \phi\left(\mathbf{X}_{s}\right) \mathbf{1}_{s}-\frac{1}{n_{t}} \phi\left(\mathbf{X}_{t}\right) \mathbf{1}_{t}\right\|_{\mathcal{H}},
$$

where $\mathbf{1}_{s}$ and $\mathbf{1}_{t}$ are the vectors with the lengths of $n_{s}$ and $n_{t}$, respectively, and their elements are all one. However, it is hard to directly learn the optimal kernel mapping operator $\phi$. To solve this problem, we relax MMD in Eq. (4) to the following formulation to serve as $f_{G}\left(\mathbf{X}_{s}, \mathbf{X}_{t}\right)$ for DR framework:

$$
\begin{aligned}
f_{G}\left(\mathbf{X}_{s}, \mathbf{X}_{t}\right) & \\
& =\left\|\frac{1}{n_{s}} \phi(\mathbf{C})^{T} \phi\left(\mathbf{X}_{s}\right) \mathbf{1}_{s}-\frac{1}{n_{t}} \phi(\mathbf{C})^{T} \phi\left(\mathbf{X}_{t}\right) \mathbf{1}_{t}\right\|_{2}^{2} .
\end{aligned}
$$


By using the above $f_{G}\left(\mathbf{X}_{s}, \mathbf{X}_{t}\right)$, we only need to learn the optimial $\phi(\mathbf{C})$, which is feasible and consistent with the model parameter of DR framework in Eq. (3). We have following lemma to show that minimizing MMD in Eq. (4) is equivalent to minimizing the proposed $f_{G}\left(\mathbf{X}_{s}, \mathbf{X}_{t}\right)$ in Eq. (5), which can support our relaxation.

Lemma 1. For $\operatorname{MMD}\left(\mathbf{X}_{s}, \mathbf{X}_{t}\right)$ and $f_{G}\left(\mathbf{X}_{s}, \mathbf{X}_{t}\right)$ defined as Eqs. (4) and (5) based on the kernel mapping operator $\phi$, we have $f_{G}\left(\mathbf{X}_{s}, \mathbf{X}_{t}\right) \rightarrow 0$ if $\operatorname{MMD}\left(\mathbf{X}_{s}, \mathbf{X}_{t}\right) \rightarrow 0$.

Proof. From the condition that $\operatorname{MMD}\left(\mathbf{X}_{s}, \mathbf{X}_{t}\right)$ is close to 0 , we know that $\phi\left(\mathbf{X}_{s}\right)$ and $\phi\left(\mathbf{X}_{t}\right)$ have similar expectations, which can be formulated as $\mathbb{E}\left(\phi\left(\mathbf{x}_{s}^{i}\right)\right) \approx \mathbb{E}\left(\phi\left(\mathbf{x}_{t}^{i}\right)\right)$, where $\mathbb{E}(\cdot)$ denotes the expectation operator. Then according to the linear property of expectation [43], i.e., $\mathbb{E}(A x)=A \mathbb{E}(x)$, it is easy to deduce that $\mathbb{E}\left(\phi(\mathbf{C})^{T} \phi\left(\mathbf{x}_{s}^{i}\right)\right) \approx \mathbb{E}\left(\phi(\mathbf{C})^{T} \phi\left(\mathbf{x}_{t}^{i}\right)\right)$, which guarantees $f_{G}\left(\mathbf{X}_{s}, \mathbf{X}_{t}\right)$ will be close to 0 .

By substituting the proposed $f_{G}\left(\mathbf{X}_{s}, \mathbf{X}_{t}\right)$ in Eq. (5) into the DR framework in Eq. (3), the optimization problem of DR framework becomes as follows:

$$
\begin{aligned}
\min _{\phi(\mathbf{C})}\left\|\mathbf{R}_{\mathcal{D}}-\phi(\mathbf{C})^{T} \phi\left(\mathbf{X}_{\mathcal{D}}\right)\right\|_{F}^{2} \\
\quad+\lambda\left\|\frac{1}{n_{s}} \phi(\mathbf{C})^{T} \phi\left(\mathbf{X}_{s}\right) \mathbf{1}_{s}-\frac{1}{n_{t}} \phi(\mathbf{C})^{T} \phi\left(\mathbf{X}_{t}\right) \mathbf{1}_{t}\right\|_{2}^{2} .
\end{aligned}
$$

Subsequently, let $\phi(\mathbf{C})=\left[\phi\left(\mathbf{X}_{s}\right), \phi\left(\mathbf{X}_{t}\right)\right] \mathbf{P}$, where $\mathbf{P} \in$ $\mathbb{R}^{\left(n_{s}+n_{t}\right) \times r}$ and $r$ is the dimension of the predefined subspace. Then, by using the kernel trick, the optimization problem of DR framework can be converted to the following formulation:

$$
\begin{aligned}
\min _{\mathbf{P}} & \left\|\mathbf{R}_{\mathcal{D}}-\mathbf{P}^{T} \mathbf{K}_{\mathcal{D}}\right\|_{F}^{2} \\
& +\lambda\left\|\frac{1}{n_{s}} \mathbf{P}^{T} \mathbf{K}_{s} \mathbf{1}_{s}-\frac{1}{n_{t}} \mathbf{P}^{T} \mathbf{K}_{t} \mathbf{1}_{t}\right\|_{2}^{2}+\mu\|\mathbf{P}\|_{1},
\end{aligned}
$$

where $\mathbf{K}_{\mathcal{D}}$ denotes the source kernel matrix $\mathbf{K}_{s}=$ $\left[\mathbf{K}_{s s}^{T}, \mathbf{K}_{s t}^{T}\right]^{T}$ or target kernel matrix $\mathbf{K}_{t}=\left[\mathbf{K}_{t s}^{T}, \mathbf{K}_{t t}^{T}\right]^{T}$ corresponding to the desired regenerated source samples $\mathbf{R}_{s}$ or target samples $\mathbf{R}_{t}$. Herein $\mathbf{K}_{s s}=\phi\left(\mathbf{X}_{s}\right)^{T} \phi\left(\mathbf{X}_{s}\right)$, $\mathbf{K}_{s t}=\phi\left(\mathbf{X}_{s}\right)^{T} \phi\left(\mathbf{X}_{t}\right), \mathbf{K}_{t s}=\phi\left(\mathbf{X}_{t}\right)^{T} \phi\left(\mathbf{X}_{s}\right)$ and $\mathbf{K}_{t t}=$ $\phi\left(\mathbf{X}_{t}\right)^{T} \phi\left(\mathbf{X}_{t}\right)$, and they can be computed by the kernel functions. This is the final formulation of the proposed DR framework. Note that in Eq. (7), we also introduce $l_{1}$ norm with respect to $\mathbf{P}$, i.e., $\|\mathbf{P}\|_{1}=\sum_{i=1}^{r}\left\|\mathbf{p}_{i}\right\|_{1}$, where $\mathbf{p}_{i}$ is the $i^{\text {th }}$ column of $\mathbf{P}$, for DR framework to serve as the regularization term. There are two important reasons. Firstly, it can avoid the overfitting problem [44] during optimizing DR framework. Secondly, each column of $\phi(\mathbf{C})$ will be enforced to reconstruct by $\phi\left(\mathbf{X}_{s}\right)$ and $\phi\left(\mathbf{X}_{t}\right)$ sparsely, which is more reasonable. The sparsity of $\mathbf{P}$ is controlled by the trade-off parameter $\mu$.

\section{Domain Regenerators under the DR Framework}

Theoretically, the predefined subspace for DR framework can be an arbitrary one. However, due to the limited useful information that are available for us in the unsupervised crossdatabase micro-expression recognition problem, we have to adequately utilize these available information to determine the predefined subspace. In other word, we should assign a
TABLE I

The Detail of the Domain Regenerators under the Proposed DR FRAMEWORK.

\begin{tabular}{|l|c|c|c|}
\hline Domain Regenerator & $\mathbf{R}_{\mathcal{D}}$ & $\mathbf{X}_{\mathcal{D}}$ in Eq. (3) & $\mathbf{K}_{\mathcal{D}}$ in Eq. (7) \\
\hline \hline DRFS-S & $\mathbf{X}_{s}$ & $\mathbf{X}_{s}$ & $\mathbf{K}_{s}$ \\
\hline DRFS-T & $\mathbf{X}_{t}$ & $\mathbf{X}_{t}$ & $\mathbf{K}_{t}$ \\
\hline DRLS & $\mathbf{L}_{s}$ & $\mathbf{X}_{s}$ & $\mathbf{K}_{s}$ \\
\hline
\end{tabular}

suitable $\mathbf{R}_{\mathcal{D}}$ for DR framework of Eq. (7) to learn an effective domain regenerator based on the given useful information. For this reason, we select two types of meaningful subspace for the proposed DR framework to perform domain renegeration. One is the original feature space and the other one is the label space which is spanned according to the label information provided by source domain. When using the original feature space as the predefined subspace for DR framework, we design two domain regenerators, i.e., (a) Domain Regeneration in the original Feature space with unchanged Source domain (DRFSS), and (b) Domain Regeneration in the original Feature space with unchanged Target domain (DRFS-T). When the label space is served for domain regeneration, we have a domain regenerator called (c) Domain Regeneration in the Label space (DRLS). Fig. 1 can provide a good example to illustrate the regenerating manners of these three domain regenerators. We summarize the employed $\mathbf{R}_{\mathcal{D}}$ and its corresponding $\mathbf{K}_{\mathcal{D}}$ in Eq. (7) (associated with $\mathbf{X}_{\mathcal{D}}$ in Eq. (3)) of the above three domain regenerators in Table I and subsequently we will introduce them in detail, respectively.

1) DRFS-S: According to Table I, we can obtain the optimization problem of DRFS-S, which can be formulated as follows:

$$
\begin{aligned}
\min _{\mathbf{P} \in \mathbb{R}^{\left(n_{s}+n_{t}\right) \times d}} & \left\|\mathbf{X}_{s}-\mathbf{P}^{T} \mathbf{K}_{s}\right\|_{F}^{2} \\
& +\lambda\left\|\frac{1}{n_{s}} \mathbf{P}^{T} \mathbf{K}_{s} \mathbf{1}_{s}-\frac{1}{n_{t}} \mathbf{P}^{T} \mathbf{K}_{t} \mathbf{1}_{t}\right\|_{2}^{2}+\mu\|\mathbf{P}\|_{1} .
\end{aligned}
$$

DRFS-S performs the regeneration in the original feature space. It is interesting to see that DRFS-S enforces the regenerated source samples to be themselves and then makes the regenerated target samples abide by the same or similar feature distributions with the original source samples. It is also worthy to mention that DRFS-S is actually the TSRG model proposed in our preliminary work of [8].

2) DRFS-T: Similar with DRFS-S, the predefined subspace for domain regeneration in DRFS-T is the original feature space. The only difference between them is that after domain regeneration, DRFS-S chooses the source domain as the unchanged domain, while DRFS-T enforces the target domain to be unchanged. The optimization problem of DRFS-T can be expressed as below:

$$
\begin{aligned}
\min _{\mathbf{P} \in \mathbb{R}^{\left(n_{s}+n_{t}\right) \times d}} & \left\|\mathbf{X}_{t}-\mathbf{P}^{T} \mathbf{K}_{t}\right\|_{F}^{2} \\
& +\lambda\left\|\frac{1}{n_{s}} \mathbf{P}^{T} \mathbf{K}_{s} \mathbf{1}_{s}-\frac{1}{n_{t}} \mathbf{P}^{T} \mathbf{K}_{t} \mathbf{1}_{t}\right\|_{2}^{2}+\mu\|\mathbf{P}\|_{1},
\end{aligned}
$$

3) DRLS: Different from DRFS-S and DRFS-T, DRLS uses the label space as the predefined subspace for domain regeneration. Due to the unsupervised problem setting, only the 
label information of source domain is available. Consequently, we can use the domain regenerator to bridge the relationship between the source domain and a label space spanned by the provided source label information. Under this consideration, let $\mathbf{R}_{\mathcal{D}}=\mathbf{L}_{s}$ and $\mathbf{K}_{\mathcal{D}}=\mathbf{K}_{s}$ and we then arrive at the optimization problem of DRLS domain regenerator:

$$
\begin{aligned}
\min _{\mathbf{P} \in \mathbb{R}^{\left(n_{s}+n_{t}\right) \times c}}\left\|\mathbf{L}_{s}-\mathbf{P}^{T} \mathbf{K}_{s}\right\|_{F}^{2} \\
+\lambda\left\|\frac{1}{n_{s}} \mathbf{P}^{T} \mathbf{K}_{s} \mathbf{1}_{s}-\frac{1}{n_{t}} \mathbf{P}^{T} \mathbf{K}_{t} \mathbf{1}_{t}\right\|_{2}^{2}+\mu\|\mathbf{P}\|_{1},
\end{aligned}
$$

\section{Optimization of DR Framework}

The optimization problem of DR framework based models can be easily solved by various methods such as iterative thresholding (IT) [17], accelerated proximal gradient (APG) [45], exact augmented Lagrange multiplier (EALM) [46] and inexact ALM (IALM) [46]. In this paper, we employ IALM method to learn the optimial $\mathbf{P}$ of DR framework based models. For convenience, we describe the detailed solving procedures with generalized formulation of DR framework in Eq. (7) as the example. More specifically, we introduce a new variable $\mathbf{Q}$ which equals $\mathbf{P}$ and then convert DR optimization problem of Eq. (7) to a constrained one as follows:

$$
\begin{aligned}
\min _{\mathbf{P}, \mathbf{Q}}\left\|\mathbf{R}_{\mathcal{D}}-\mathbf{Q}^{T} \mathbf{K}_{\mathcal{D}}\right\|_{F}^{2} \\
\quad+\lambda\left\|\frac{1}{n_{s}} \mathbf{Q}^{T} \mathbf{K}_{s} \mathbf{1}_{s}-\frac{1}{n_{t}} \mathbf{Q}^{T} \mathbf{K}_{t} \mathbf{1}_{t}\right\|_{2}^{2}+\mu\|\mathbf{P}\|_{1}, \\
\text { s.t. } \mathbf{P}=\mathbf{Q}, \quad(\mathcal{D} \in\{s, t\}) .
\end{aligned}
$$

Subsequently, the Lagrange function can be obtained as the following formulation:

$$
\begin{array}{r}
L(\mathbf{P}, \mathbf{Q}, \mathbf{T}, \kappa)=\left\|\mathbf{R}_{\mathcal{D}}-\mathbf{Q}^{T} \mathbf{K}_{\mathcal{D}}\right\|_{F}^{2}+\lambda\left\|\mathbf{Q}^{T} \Delta \mathbf{k}_{s t}\right\|_{2}^{2} \\
+\mu\|\mathbf{P}\|_{1}+\operatorname{tr}\left[\mathbf{T}^{T}(\mathbf{P}-\mathbf{Q})\right]+\frac{\kappa}{2}\|\mathbf{P}-\mathbf{Q}\|_{F}^{2}
\end{array}
$$

where $\Delta \mathbf{k}_{s t}=\frac{1}{n_{s}} \mathbf{K}_{s} \mathbf{1}_{s}-\frac{1}{n_{t}} \mathbf{K}_{t} \mathbf{1}_{t}$.

Finally, to learn the optimal $\mathbf{P}$, we only need to minimize the Lagrange function of Eq. (12) with respect to one of the variables while fixing the others iteratively. Specifically, repeating the following four steps until obtaining convergence:

1) Fix $\mathbf{P}, \mathbf{T}, \kappa$ and update $\mathbf{Q}$ : In this step, the optimization problem with respect to $\mathbf{Q}$ is as follows:

$$
\begin{aligned}
\min _{\mathbf{Q}} & \left\|\mathbf{R}_{\mathcal{D}}-\mathbf{Q}^{T} \mathbf{K}_{\mathcal{D}}\right\|_{F}^{2}+\lambda\left\|\mathbf{Q}^{T} \Delta \mathbf{k}_{s t}\right\|_{2}^{2}+\operatorname{tr}\left[\mathbf{T}^{T}(\mathbf{P}-\mathbf{Q})\right] \\
& +\frac{\kappa}{2}\|\mathbf{P}-\mathbf{Q}\|_{F}^{2},
\end{aligned}
$$

whose close-form solution is

$$
\mathbf{Q}=\left(\mathbf{K}_{\mathcal{D}} \mathbf{K}_{\mathcal{D}}^{T}+\Delta \mathbf{k}_{s t} \Delta \mathbf{k}_{s t}^{T}+\frac{\kappa \mathbf{I}}{2}\right)^{-1}\left(\mathbf{K}_{\mathcal{D}} \mathbf{R}_{\mathcal{D}}^{T}+\frac{\kappa \mathbf{P}+\mathbf{T}}{2}\right),
$$

2) Fix $\mathbf{Q}, \mathbf{T}, \kappa$ and update $\mathbf{P}$ :

$$
\min _{\mathbf{P}} \frac{\mu}{\kappa}\|\mathbf{P}\|_{1}+\frac{1}{2}\left\|\mathbf{P}-\left(\mathbf{Q}-\frac{\mathbf{T}}{\kappa}\right)\right\|_{F}^{2} .
$$

By using the soft-thresholding operator, we are able to obtain the optimal $\mathbf{P}$ according to the following criterion:

$$
\mathbf{P}_{i j}= \begin{cases}\left(\mathbf{Q}_{i j}-\frac{\mathbf{T}_{i j}}{\kappa}\right)-\frac{\mu}{\kappa}, & \text { if }\left(\mathbf{Q}_{i j}-\frac{\mathbf{T}_{i j}}{\kappa}\right)>\frac{\mu}{\kappa} ; \\ \left(\mathbf{Q}_{i j}-\frac{\mathbf{T}_{i j}}{\kappa}\right)+\frac{\mu}{\kappa}, & \text { if }\left(\mathbf{Q}_{i j}-\frac{\mathbf{T}_{i j}}{\kappa}\right)<\frac{\mu}{\kappa} ; \\ 0, \quad \text { otherwise, } & \end{cases}
$$

where $\mathbf{P}_{i j}, \mathbf{Q}_{i j}$, and $\mathbf{T}_{i j}$ are the elements in the $i^{t h}$ row and $j^{\text {th }}$ column of their corresponding matrices.

3) Update $\mathbf{T}$ and $\kappa$ :

$$
\mathbf{T}=\mathbf{T}+\kappa(\mathbf{P}-\mathbf{Q}), \kappa=\min \left(\rho \kappa, \kappa_{\max }\right),
$$

where $\rho$ is a scaled parameter.

4) Check convergence:

$$
\|\mathbf{P}-\mathbf{Q}\|_{\infty}<\epsilon,
$$

where $\epsilon$ denotes the machine epsilon.

\section{EXPERIMENTS}

\section{A. Micro-Expression Databases and Experiment Protocol}

In this section, we conduct extensive cross-database microexpression recognition experiments to evaluate the performance of the proposed DR framework. Two publicly available micro-expression databases are used. One is SMIC database, which is built by Li et al. [47]. SMIC database consists of three types of datasets, i.e., SMIC (HS), SMIC (VIS), and SMIC (NIR), which are recorded by a high speed (HS) camera of $100 \mathrm{fps}$, a normal visual (VIS) camera of 25fps, and a near-infrared (NIR) camera, respectively. SMIC (HS) contains 164 micro-expression clips from 16 different subjects, while both SMIC (VIS) and SMIC (NIR) consist of 71 samples from 8 participants. The samples of three datasets of SMIC are all divided into three micro-expression categories, i.e., Positive, Negative, and Surprise. CASME II database is collected by Yan et al. [48]. It includes 26 subjects and records their 247 micro-expression samples. These samples are categorized into five micro-expression classes including Happiness, Surprise, Disgust, Repression, and Others, respectively. In this paper, following our previous work [12], the face images of the video clips from CASME II database are cropped and then transformed to $308 \times 257$ pixels, while for the samples from three SMIC datasets, we crop and transform the images into $170 \times 139$ pixels for experiments. We select an image frame from the micro-expression video clips belonging to the above four datasets, respectively, which are shown in Fig. 2, to serve as the examples such that the readers can glance through the difference among CASME II and three datasets of SMIC.

We design two types of cross-database micro-expression recognition experiments in this paper. The first type of experiments (denoted by TYPE-I) are between CASME II and one dataset of SMIC (HS, VIS, NIR), i.e., CASME II v.s. SMIC (HS), CASME II v.s. SMIC (VIS), and CASME II v.s. SMIC (NIR). The second type of experiments (denoted by TYPEII) are between either two datasets of SMIC (HS, VIS, NIR) database including HS v.s. VIS, HS v.s. NIR, and VIS v.s. NIR. Since the two employed datasets in each of the above 


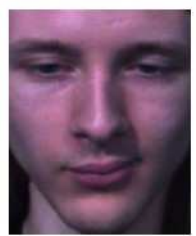

(a) SMIC (HS)

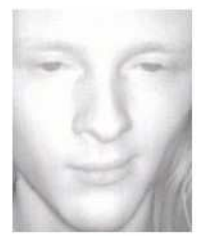

(b) SMIC (NIR)

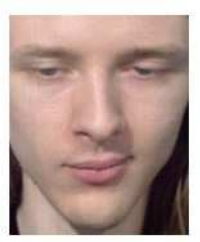

(c) SMIC (VIS)

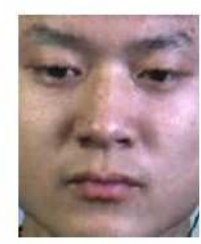

(d) CASME II
Fig. 2. Examples of SIMC and CASME II micro-expression databases. From left to right, they are image frames of the micro-expression video clips from (a) SMIC (HS), (b) SMIC (NIR), (c) SMIC (VIS), and (d) CASME II, respectively.

combinations are alternatively served as source and target databases, there are six groups in each type of experiments. For convenience, we denote the total 12 experiments by Exp.1, $\cdots$, Exp.6 (TYPE-I) and Exp.7, $\cdots$, Exp.12 (TYPE-II), respectively. In order to make CASME II and three datasets of SMIC share the same micro-expression categories, we select the samples belonging to Happiness, Surprise, Disgust, and Repression from CASME II and re-label these samples. Specifically, the samples of Happiness are given Positive labels and the samples of Disgust and Repression are categorized into Negative. The labels of Surprise samples are unchanged.

The new sample information of SMIC (HS, VIS, NIR) and CASME II with respect to consistent micro-expression categorization is shown in Table II. From Table II, it can be seen that class imbalance problem exists in CASME II and SMIC (HS) datasets, which means the number of one type of micro-expression samples is significantly larger or lower than other types of micro-expression samples. Consequently, for better reporting the experimental results, we choose two metrics widely used in cross-database speech emotion recognition, i.e., unweighted average recall (UAR) and weighted average recall (WAR) [29], in the experiments. According to the definition in [29], WAR is the normal recognition accuracy, while UAR is the mean accuracy of each class divided by the number of classes without consideration of samples per class. It can comprehensively reveal the true performance of one method by comparing the WAR and UAR of this method. For example, if there is a big gap between a high WAR and a low UAR in a method, it usually occurs that most of target samples are predicted by this method as the micro-expression category whose sample number percentage is dominant among all the micro-expression samples. Consequently, this method cannot be deemed to perform good even though it achieves a high WAR (recognition accuracy).

For comparison, some recently proposed well-performing cross-database emotion (speech emotion and facial expression) recognition methods including KMM [30], [39], KLIEP [30], [40], uLSIF [30], [41], and STM [24], [25] are chosen. Note that SVM is served as the classifier for all the methods. Besides, SVM without any domain adaptation is also included for comparison to serve as a baseline method. The detailed parameter setting of all the methods and micro-expression features employed in the experiments are as follows:
TABLE II

THE SAMPLE INFORMATION OF SMIC (HS, VIS, NIR) AND CASME II WITH RESPECT TO CONSISTENT MICRO-EXPRESSION CATEGORIZATION.

\begin{tabular}{|l|c|c|c|}
\hline \multirow{2}{*}{ Dataset } & \multicolumn{3}{|c|}{ Micro-Expression Category } \\
\cline { 2 - 4 } & Negative & Positive & Surprise \\
\hline \hline CASME II & 91 & 32 & 25 \\
\hline SMIC (HS) & 70 & 51 & 43 \\
\hline SMIC (VIS) & 28 & 23 & 20 \\
\hline SMIC (NIR) & 28 & 23 & 20 \\
\hline
\end{tabular}

1) For micro-expression feature, we use uniform LBPTOP [9] and the neighboring radius $R$ and the number of the neighboring points $P$ for LBP operator on three orthogonal planes are set as 3 and 8 , respectively. Besides, following the work of [49], a multi-scale spatial division scheme consisting of $1 \times 1,2 \times 2,4 \times 4$, and $8 \times 8$ grids is adopted to partition the micro-expression video clips into a few facial blocks. Consequently, each micro-expression sample is described by a feature vector comprising the LBP-TOP vectors of all the facial blocks.

2) We use linear kernel function and set $C=1$ for SVM in the experiments. Meanwhile, for fair comparison, linear kernel function is adopted for all the methods throughout the experiments.

3) For KMM, according to the suggestion of [39], its two important parameters including the upper limit of importance weight $B$ and $\epsilon$ are set as 1000 and $\sqrt{n_{t r}}-\frac{1}{\sqrt{n_{t r}}}$, where $n_{t r}$ denotes the number of training samples. For STM, following the work of [24], [25], the upper limit of importance weight $B$ and $\epsilon$ are set as the same values with those of KMM.

4) For KLIEP, no parameter needs be set, while for ULSIF, STM, and the proposed DR framework based methods (DRFS-S, DRFS-T, and DRLS), there are tradeoff parameters to be set. Since the label information of target domain is entirely unknown, cross-validation method is not available for determining the trade-off parameters. Consequently, to offer a fair comparison among all the methods, in the experiments we use the parameter grid search strategy for these methods and report the best results which correspond to the optimal trade-off parameters. The optimal trade-off parameters for all the methods used in different experiments are given in Appendix A.

\section{B. Experimental Results and Analysis}

The WAR and UAR of TYPE-I and TYPE-II experiments obtained by all the methods are shown in Tables III, IV, V, and VI, respectively. From these Tables, it is clear that nearly in all the experiments, three domain regenerators under the proposed DR framework achieve promising increases in the performance over SVM without domain adaptation. Moreover, our method achieves the best WAR and UAR in NINE of 12 cases including Exp.1 (DRLS), Exp.3 (DRFS-S), Exp.4, Exp.5 (DRLS), Exp.7 (DRLS), Exp.8 (DRFS-T), Exp.10 (DRFS-T), Exp.11 (DRFS-T), and Exp.12 (DRFS-S), where the domain 
TABLE III

EXPERIMENTAL RESULTS ON CASME II AND ONE SUBSET OF SMIC (HS, VIS, NIR) DATABASES IN RECOGNIZING THREE MICRO-EXPRESSIONS

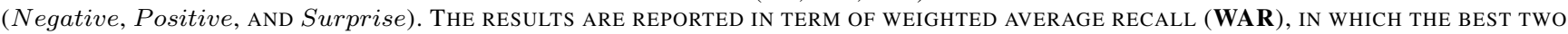
RESULTS ARE HIGHLIGHTED IN BOLD.

\begin{tabular}{|c|c|c|c|c|c|c|c||c|c|c|}
\hline \multirow{2}{*}{ Exp. } & \multicolumn{1}{|c|}{ Micro-Expression Database } & \multicolumn{9}{|c|}{ Method } \\
\cline { 2 - 12 } & Source & Target & SVM & KMM & KLIEP & uLSIF & STM & DRFS-S & DRFS-T & DRLS \\
\hline \hline 1 & CASME II & SMIC (HS) & 42.07 & 37.20 & 45.73 & 47.56 & 46.34 & $\mathbf{5 0 . 6 1}$ & 47.56 & $\mathbf{5 4 . 8 8}$ \\
\hline 2 & SMIC (HS) & CASME II & 24.32 & 27.70 & 24.32 & $\mathbf{6 6 . 2 2}$ & 58.11 & 64.19 & $\mathbf{6 4 . 8 6}$ & 62.16 \\
\hline \hline 3 & CASME II & SMIC (VIS) & 45.07 & 26.76 & 45.07 & 54.93 & $\mathbf{5 6 . 3 4}$ & $\mathbf{6 0 . 5 6}$ & 42.25 & 53.52 \\
\hline 4 & SMIC (VIS) & CASME II & 36.49 & 29.73 & 36.49 & 39.19 & $\mathbf{5 1 . 3 5}$ & $\mathbf{5 2 . 0 3}$ & 50.03 & 49.32 \\
\hline \hline 5 & CASME II & SMIC (NIR) & 45.07 & 26.76 & 47.89 & $\mathbf{5 0 . 7 0}$ & 46.48 & 45.07 & 40.85 & $\mathbf{5 6 . 3 4}$ \\
\hline 6 & SMIC (NIR) & CASME II & 16.22 & 21.62 & 16.22 & 29.05 & $\mathbf{6 0 . 8 1}$ & 42.57 & 43.24 & $\mathbf{4 3 . 9 2}$ \\
\hline
\end{tabular}

TABLE IV

EXPERIMENTAL RESULTS ON CASME II AND ONE SUBSET OF SMIC (HS, VIS, NIR) DATABASES IN RECOGNIZING THREE MICRO-EXPRESSIONS (Negative, Positive, AND Surprise). THE RESUlts ARE REPORTED IN TERM OF UNWEIGHTED AVERAGE RECALl (UAR), IN WHICH THE BEST TWO RESULTS ARE HIGHLIGHTED IN BOLD.

\begin{tabular}{|c|c|c|c|c|c|c|c||c|c|c|}
\hline \multirow{2}{*}{ Exp. } & Micro-Expression Database & \multicolumn{9}{|c|}{ Method } \\
\cline { 2 - 12 } & Source & Target & SVM & KMM & KLIEP & uLSIF & STM & DRFS-S & DRFS-T & DRLS \\
\hline \hline 1 & CASME II & SMIC (HS) & 37.91 & 34.66 & 42.32 & 47.91 & $\mathbf{4 9 . 6 4}$ & 47.48 & 42.37 & $\mathbf{5 4 . 6 5}$ \\
\hline 2 & SMIC (HS) & CASME II & 35.14 & 39.67 & 35.14 & 43.43 & 41.17 & $\mathbf{4 9 . 5 8}$ & $\mathbf{4 7 . 1 4}$ & 41.24 \\
\hline \hline 3 & CASME II & SMIC (VIS) & 42.67 & 25.72 & 41.93 & $\mathbf{5 3 . 1 2}$ & 53.10 & $\mathbf{5 8 . 9 6}$ & 43.01 & 52.49 \\
\hline 4 & SMIC (VIS) & CASME II & 48.94 & 44.02 & 49.91 & 53.31 & 39.99 & $\mathbf{5 6 . 0 3}$ & 51.29 & $\mathbf{5 7 . 6 5}$ \\
\hline \hline 5 & CASME II & SMIC (NIR) & 43.67 & 23.14 & 45.58 & $\mathbf{5 1 . 5 1}$ & 41.27 & 45.80 & 39.70 & $\mathbf{5 6 . 9 5}$ \\
\hline 6 & SMIC (NIR) & CASME II & 31.42 & 36.54 & 31.71 & 40.26 & 34.32 & $\mathbf{4 3 . 9 4}$ & 37.93 & $\mathbf{4 6 . 8 9}$ \\
\hline
\end{tabular}

regenerator in brackets behind Exp. obtains both highest WAR and UAR in the corresponding experiment. It is also interesting to see that among three domain regenerators, DRLS seems most suitable for TYPE-I experiments, while DRFS-T is best at dealing with TYPE-II experiments. Additionally, it is clear to see that ULSIF outperforms the proposed DR framework in terms of both WAR and UAR in Exp. 9 and in term of WAR in Exp.2, which shows its better effectiveness than other methods. However, we can find that some of our DR based methods are in fact very competitive against ULSIF in these two cases.

Furthermore, it can be found that all the methods can achieve overall better results in TYPE-I experiments than TYPE-II experiments, which indicates that compared with TYPE-II, TYPE-I experiments are really more challenging. In other words, we are able to conclude that the difference between CASME II and SMIC is larger than the difference among three datasets of SMIC. In fact, according to the works of [47] and [48], it can be obtained that the stimuli materials, the race and age of participants, and the equipment and environments used for building SMIC and CASME II databases are very different, which exacerbates the sample difference existing between CASME II and SMIC databases. On the other hand, from the comparison between Exps.3 and 4 and Exps.5 and 6 and the comparison between Exps.7 and 8 and Exps.9 and 10, it is interesting to see that no matter SMIC (NIR) is served as source or target database, the experiments involving SMIC (NIR) are more difficult than the experiments of employing SMIC (VIS). We think this may attribute to the heterogeneous problem between NIR images and CASME II or VIS images. As Fig. 2 shows, NIR images have large quality difference from CASME II or VIS images.
Besides, one more interesting finding can be obtained according to the two experimental results in each group of dataset combination (e.g., Exp.1 and Exp. 2 using CASME II and SMIC (HS)). Compared with the experiments of using CASME II or SMIC (HS) as source database, the experimental results of using CASME II or SMIC (HS) as target database are obviously at lower levels. For example, it is clear that the average WAR and UAR of all the methods in Exp.8, where SMIC (HS) is served as target database, is much less than that in Exp.7, in which SMIC (HS) is source database. It is very likely due to the class imbalance problem, which exists in CASME II and SMIC (HS) datasets and can be seen in Table II. Especially in CASME II, the class imbalance problem is more serious and the number of Negative samples is even larger than the number summation of Positive and Surprise samples. Additionally, we also observe that compared with the experiments of using SMIC (HS) as target database (Exp.1, Exp.8, and Exp.10), there is a big gap between the WAR and UAR in the experiments of using CASME II as source database (Exp.2, Exp.4, and Exp.6). It is believed that the more extreme class imbalance problem in CASME II over SMIC (HS) may be the main reason.

As a good example to demonstrate the above point, Exp.6 is a tough task among all the experiments of using CASME II as target database, in which most of methods achieve bad WAR, UAR and a large gap between WAR and UAR. Despite of this, it is surprising from the results to see that STM and the proposed three domain regenerators (DRFS-S, DRFS-T, and DRLS) achieve WAR of $60.81 \%, 42.57 \%, 43.24 \%$, and $43.92 \%$, respectively, which are considerably higher than other four comparison methods. By further comparing the WAR 
TABLE V

EXPERIMENTAL RESULTS ON TWO DATASETS OF SMIC (HS, VIS, NIR) DATABASES IN RECOGNIZING THREE MICRO-EXPRESSIONS ( Negative, Positive, AND Surprise). THE RESUlts ARE REPORTED IN TERM OF WEIGHTED AVERAGE RECALl (WAR), IN WHICH THE BEST TWO RESUlTS ARE HIGHLIGHTED IN BOLD.

\begin{tabular}{|c|c|c|c|c|c|c|c||c|c|c|}
\hline \multirow{2}{*}{ Exp. } & \multicolumn{2}{|c|}{ Micro-Expression Database } & \multicolumn{9}{|c|}{ Method } \\
\cline { 2 - 12 } & Source & Target & SVM & KMM & KLIEP & uLSIF & STM & DRFS-S & DRFS-T & DRLS \\
\hline \hline 7 & HS & VIS & 78.87 & 81.69 & 78.87 & 85.92 & $\mathbf{9 0 . 1 4}$ & 88.73 & 85.92 & $\mathbf{9 1 . 5 5}$ \\
\hline 8 & VIS & HS & 59.76 & 48.17 & 59.76 & 60.98 & 61.59 & $\mathbf{6 2 . 8 0}$ & $\mathbf{6 4 . 6 3}$ & 60.37 \\
\hline \hline 9 & HS & NIR & 70.42 & 59.15 & 69.01 & $\mathbf{7 7 . 4 6}$ & $\mathbf{7 6 . 0 6}$ & 70.42 & 74.65 & 70.42 \\
\hline 10 & NIR & HS & 53.05 & 46.34 & 53.05 & 55.49 & 55.49 & $\mathbf{6 2 . 2 0}$ & $\mathbf{6 3 . 4 1}$ & 60.98 \\
\hline \hline 11 & VIS & NIR & 69.01 & 70.42 & 70.42 & $\mathbf{7 3 . 2 4}$ & 71.83 & $\mathbf{7 3 . 2 4}$ & $\mathbf{8 0 . 2 8}$ & $\mathbf{7 3 . 2 4}$ \\
\hline 12 & NIR & VIS & 69.01 & 66.20 & 67.61 & 78.87 & 76.06 & $\mathbf{8 3 . 1 0}$ & 77.46 & 81.69 \\
\hline
\end{tabular}

TABLE VI

EXPERIMENTAL RESULTS ON TWO DATASETS OF SMIC (HS, VIS, NIR) DATABASES IN RECOGNIZING THREE MICRO-EXPRESSIONS (Negative, Positive, AND Surprise). THE RESUlts ARE REPORTED IN TERM OF UNWEIGHTED AVERAGE RECALl (UAR), IN WHICH THE BEST TWO RESULTS ARE HIGHLIGHTED IN BOLD.

\begin{tabular}{|c|c|c|c|c|c|c|c||c|c|c|}
\hline \multirow{2}{*}{ Exp. } & \multicolumn{1}{|c|}{ Micro-Expression Database } & \multicolumn{10}{|c|}{ Method } \\
\cline { 2 - 13 } & Source & Target & SVM & KMM & KLIEP & uLSIF & STM & DRFS-S & DRFS-T & DRLS \\
\hline \hline 7 & HS & VIS & 79.55 & 81.06 & 79.55 & 86.32 & $\mathbf{9 0 . 1 1}$ & 88.03 & 86.88 & $\mathbf{9 0 . 9 1}$ \\
\hline 8 & VIS & HS & 62.30 & 48.90 & 62.30 & 63.85 & 63.55 & $\mathbf{6 4 . 8 8}$ & $\mathbf{6 6 . 8 4}$ & 61.72 \\
\hline \hline 9 & HS & NIR & 69.63 & 61.76 & 68.44 & $\mathbf{7 7 . 9 3}$ & 74.57 & 71.88 & $\mathbf{7 4 . 7 2}$ & 71.15 \\
\hline 10 & NIR & HS & 57.17 & 50.88 & 57.35 & 59.61 & 59.42 & $\mathbf{6 3 . 5 6}$ & $\mathbf{6 5 . 0 6}$ & 62.73 \\
\hline \hline 11 & VIS & NIR & 69.14 & 70.80 & 70.80 & 73.19 & 72.73 & $\mathbf{7 4 . 4 0}$ & $\mathbf{7 9 . 8 7}$ & 71.54 \\
\hline 12 & NIR & VIS & 69.45 & 65.60 & 68.00 & 78.64 & 76.56 & $\mathbf{8 3 . 2 5}$ & 77.97 & $\mathbf{8 1 . 3 2}$ \\
\hline
\end{tabular}

and UAR of STM and the proposed DR framework in this experiment, we can clearly find that the gap between WAR and UAR of the proposed DR framework $(42.57 \%$ and $43.94 \%$ by DRFS-S, $43.24 \%$ and $37.93 \%$ by DRFS-T, and $43.92 \%$ and $46.89 \%$ by DRLS) is much narrower than STM $(60.81 \%$ and $34.32 \%$ ). Due to the dominant percentage of Negative samples in CASME II, it is believed that most of CASME II samples may be mistakenly predicted as Negative by STM and hence the STM method achieves a low UAR although its WAR is leading among all the methods. In other words, the gap of our results is actually more acceptable, which indicates that the proposed DR framework is less affected by the extreme class imbalance problem existing in CASME II and is more applicable to this challenging experiment.

In order to check the above analysis and further see how the extreme class imbalance problem affects the experimental results of each method, we select two pairs of experiments including Exps. 3 and 4 and Exps.5 and 6, where CASME II is served as source and target database, respectively, as the representatives and draw the confusion matrices of all eight methods in these four experiments. The confusion matrices of all the methods in Exp.3, Exp.4, Exp.5 and Exp.6 are depicted in Figs. 3, 4, 5, and 6, respectively. From these confusion matrices, some interesting findings and conclusions can be obtained:

Firstly, as the confusion matrix of STM in Fig. 6 (Exp.6) shows, it is clear that nearly all the samples of CASME II database are predicted as Negative micro-expression by STM, which is consistent with our analysis previously. Consequently, the above analysis to explain why a big gap between the WAR and UAR exists in STM is reasonable.
Secondly, from the comparison between Fig. 3 (Exp.3) and Fig. 4 (Exp.4) and the comparison between Fig. 5 (Exp.5) and Fig. 6 (Exp.6), it can be found that for all the methods, three micro-expressions are much more easily confused in the case when CASME II is served as the target database (Fig. 4 (Exp.4) and Fig. 6 (Exp.6)) than the opposite case where CASME II is used as the source database (Fig. 3 (Exp.3) and Fig. 5 (Exp.5)). This indicates that if the class imbalance problem occurred in the target database, cross-database microexpression tasks would become more challenging and hence domain adaptation methods would be more possibly interfered such that their performance may be decreased.

Thirdly, by further comparing Fig. 3 (Exp.3) and Fig. 4 (Exp.4), we can also find that in the experiments of using CASME II as source database, it is easy for most of methods to mistakenly predict target samples as Negative microexpression, whose corresponding samples have a dominant percentage in CASME II. On the contrary, when CASME II is used as target database in the experiments, its Negative samples would be wrongly predicted as other micro-expressions by most of methods.

Finally, it is clear to see that even though most of comparison methods perform very poorly in the experiments of using CASME II as target database, i.e., Exp.4 (Fig. 4) and Exp.6 (Fig. 4), the proposed DR framework (DRFS-S, DRFS$\mathrm{T}$, and DRLS) can still achieve satisfactory results, in which the extreme micro-expression confusion situation occurs in other comparison methods is promisingly alleviated.

Fourthly, it can also from Fig. 6 (Exp.6) be observed that compared with Fig. 4 (Exp.4), the wrong prediction of Negative samples in CASME II in the experiments of 


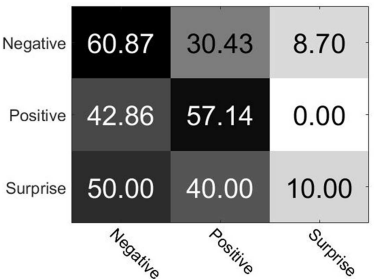

(a) SVM

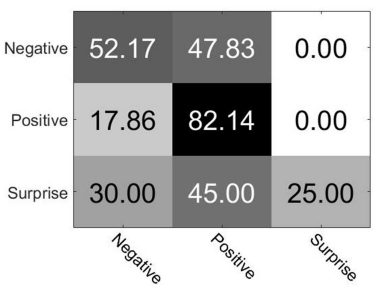

(e) STM

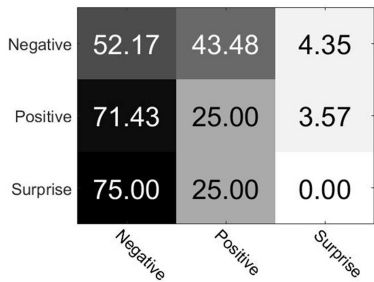

(b) KMM

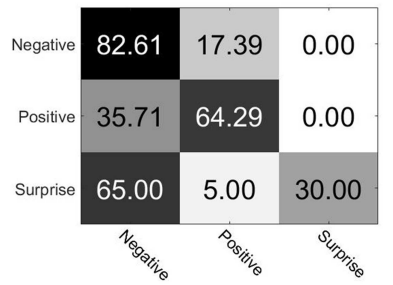

(f) DRFS-S

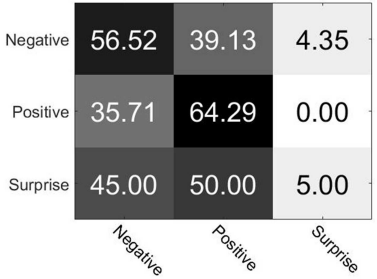

(c) KLIEP

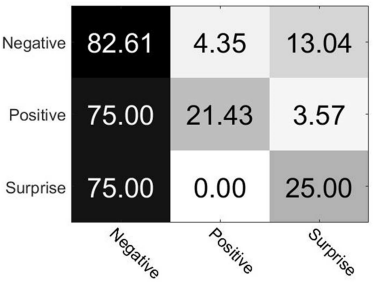

(g) DRFS-T

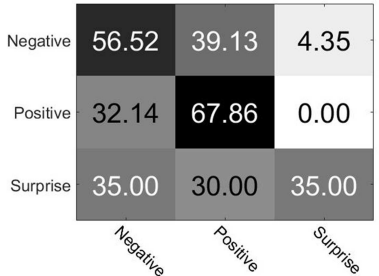

(d) ULSIF

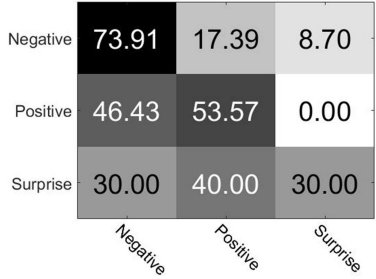

(h) DRLS

Fig. 3. The confusion matrices of all the methods in Exp.3. From (a) to (h), the results are SVM, KMM, KLIEP, STM, DRFS-S, DRFS-T, and DRLS, respectively.

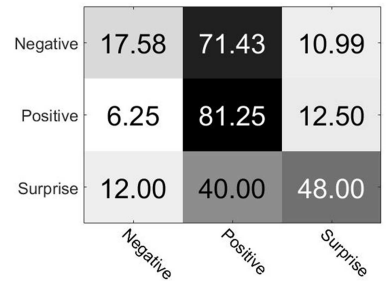

(a) SVM

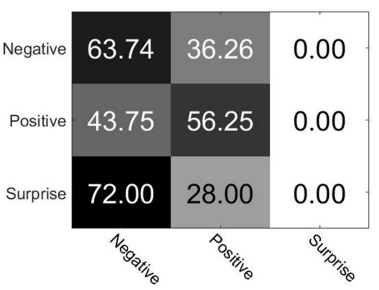

(e) STM

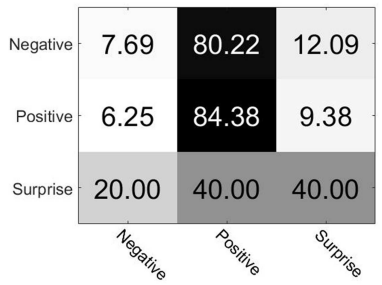

(b) KMM

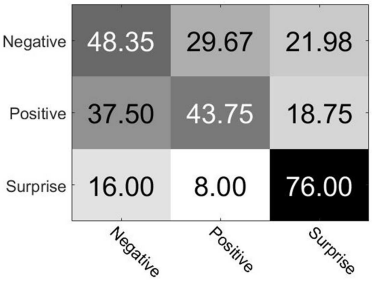

(f) DRFS-S

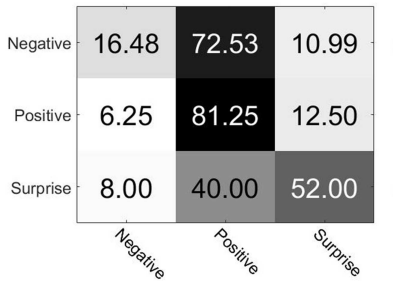

(c) KLIEP

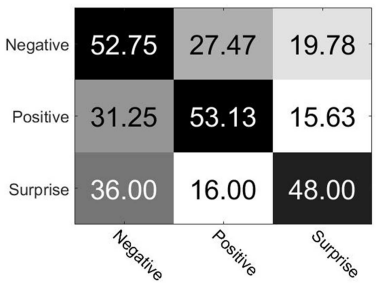

(g) DRFS-T

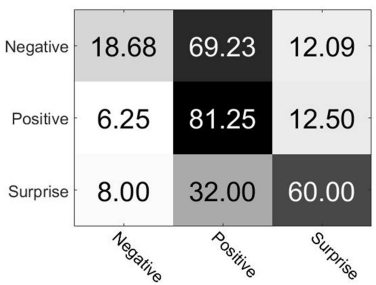

(d) ULSIF

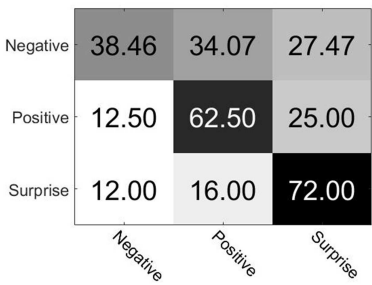

(h) DRLS

Fig. 4. The confusion matrices of all the methods in Exp.4. From (a) to (h), the results are SVM, KMM, KLIEP, STM, DRFS-S, DRFS-T, and DRLS, respectively.

using SMIC (NIR) as target database would deteriorate and the confusion between Negative and other micro-expression samples becomes extreme. As described previously, besides the class imbalance problem in CASME II, we think the heterogeneous problem of facial images between CASME II and SMIC (NIR) datasets is a major factor as well. The comparison between Fig. 5 (Exp.5) and Fig. 3 (Exp.3) can also provide a support for this viewpoint. As Fig. 5 (Exp.5) demonstrates, the confusion among three micro-expressions is much more serious than that shown in Fig. 3 (Exp.3).

\section{Comparison among Three Domain Regenerators}

From the results of DRFS-S, DRFS-T, and DRLS shown in Tables III, IV, V, and VI, we can find that these three domain regenerators have large difference in performance in some cases. This seems opposite to some intuitions. Firstly, it is intuitive that DRFS-S and DRFS-T would have same or similar performance because their definitions and motivations look so similar. Secondly, it looks that DRLS should perform better than other two domain regenrators since it utilizes more useful information, i.e., the label information provided by the source samples. In this section, we further deeply compare and discuss three proposed domain regenerators by answering the above two questions.

1) Why do DRFS-S and DRFS-T have different performance?: Intuitively, DRFS-S and DRFS-T should have similar recognition performance. In fact, there are major differences between them. The classification performance of these two DR models for the target micro-expression samples largely depends on the classifier, which is learned by whether the original or regenerated source micro-expression samples. Specifically, for the DRFS-S model, the classifier is learned based on the original source micro-expression samples and then used to classify the regenerated target micro-expression samples that 


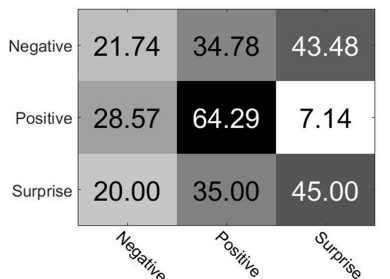

(a) SVM

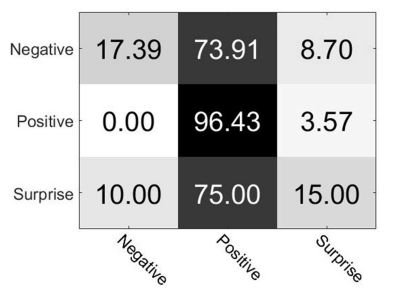

(e) STM

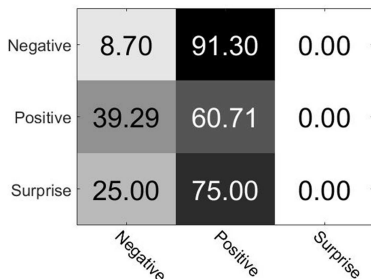

(b) KMM

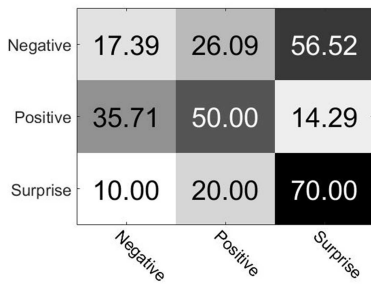

(f) DRFS-S

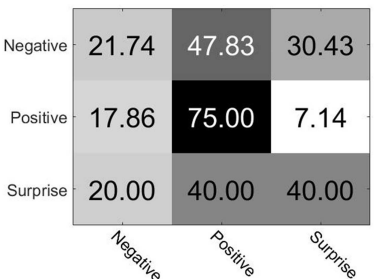

(c) KLIEP

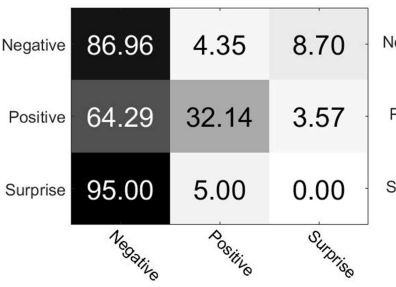

(g) DRFS-T

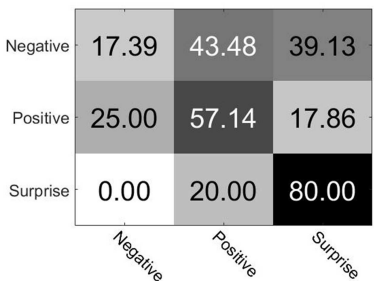

(d) ULSIF

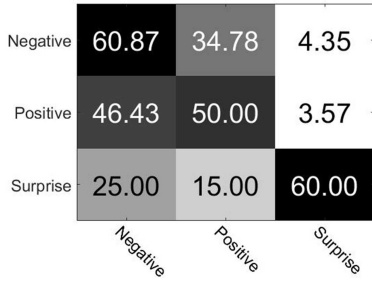

(h) DRLS

Fig. 5. The confusion matrices of all the methods in Exp.5. From (a) to (h), the results are SVM, KMM, KLIEP, STM, DRFS-S, DRFS-T, and DRLS, respectively.

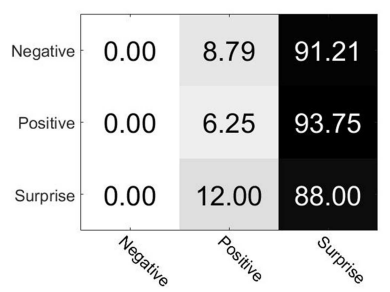

(a) SVM

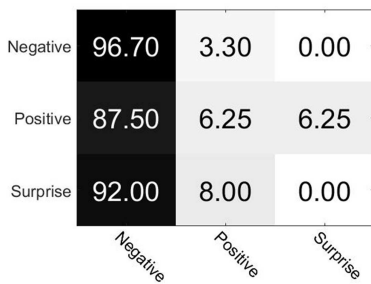

(e) STM

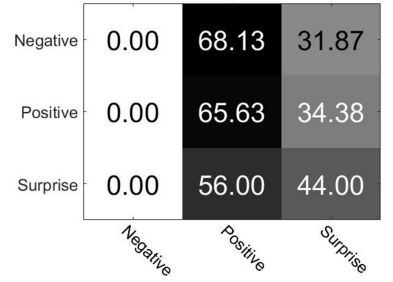

(b) KMM

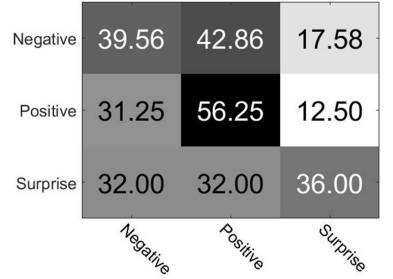

(f) DRFS-S

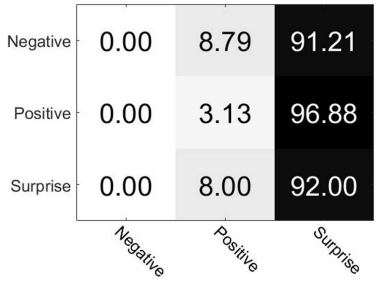

(c) KLIEP

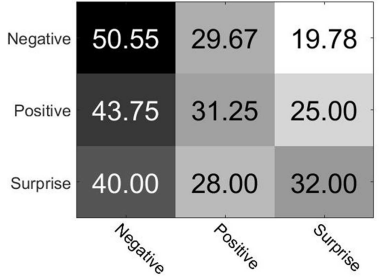

(g) DRFS-T

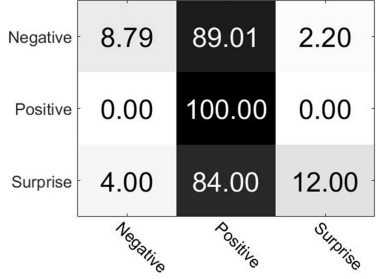

(d) ULSIF

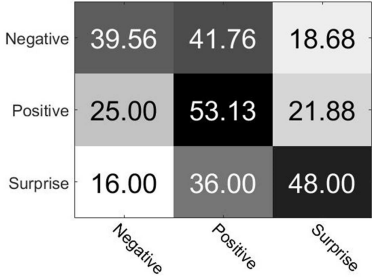

(h) DRLS

Fig. 6. The confusion matrices of all the methods in Exp.6. From (a) to (h), the results are SVM, KMM, KLIEP, STM, DRFS-S, DRFS-T, and DRLS, respectively.

share the similar distribution of the source micro-expression samples. For the DRFS-T model, however, the classifier is learned based on the regenerated source micro-expression samples, which share the similar feature distribution of the target micro-expression samples, instead of the original ones. Then, the classifier is used to classify the original target micro-expression samples. Consequently, if the source microexpression samples of different classes are well-separated, then the DRFS-S model may perform well. However, if the target micro-expression samples of different classes are wellseparated, which makes the regenerated source samples wellseparated as well, the DRFS-T would perform well.

2) Why does not DRLS always outperform DRFS-S and DRFS-T?: From the experimental results, it is clear that in some cases, DRLS does not outperform DRFS-S and DRFS-T although DRLS uses more information, i.e., the label information. This is very likely due to the fact that, in some cases, the original source micro-expression samples or the original target micro-expression samples are more separable than the corresponding ones in the label space. Consequently, the classifier learned based on source samples (corresponding to DRFS-S) or the ones regenerated by DRFS-T would achieve better performance than DRLS.

\section{Parameter Sensitivity of $D R$ framework}

The proposed DR framework has two important tradeoff parameters including $\lambda$ and $\mu$, whose selection affects the performance of DR framework. In the experiments, we just employ the fixed trade-off parameters and hence it is easy to bring a question whether the performance of DR framework would be sensitive to the slight changes of such 


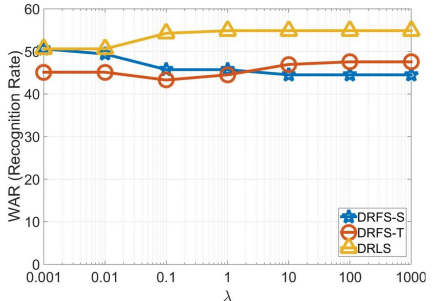

(a) Exp.1

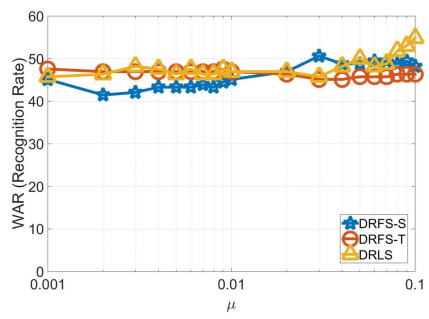

(e) Exp.1

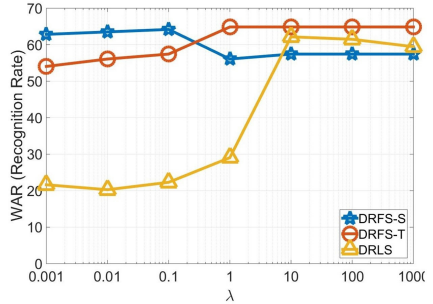

(b) Exp.2

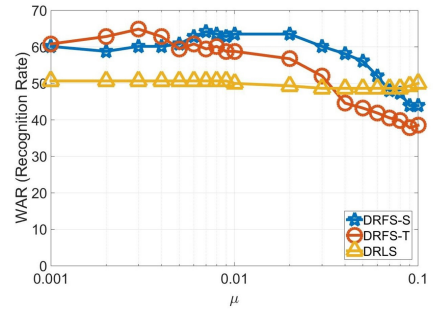

(f) Exp. 2

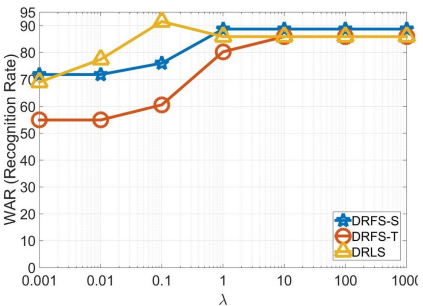

(c) Exp.7

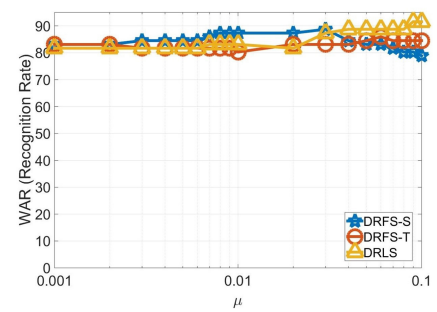

(g) Exp.7

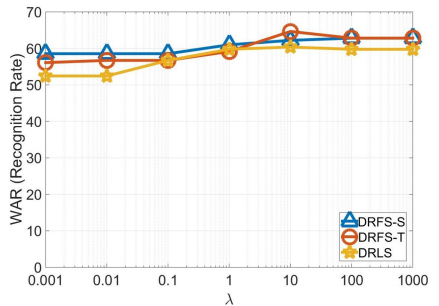

(d) Exp.8

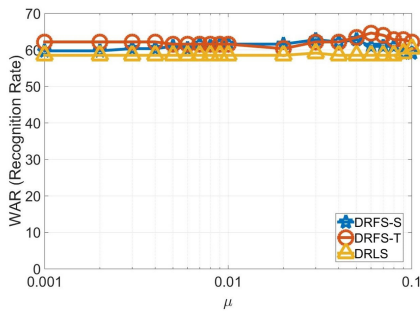

(h) Exp.8

Fig. 7. The results of trade-off parameter sensitive experiments for the proposed DR framework. Exp.1 corresponds to (a) and (e), Exp. 2 corresponds to (b) and (f), Exp.7 corresponds to (c) and (g), and Exp.8 corresponds to (d) and (h), respectively. In this picture, the top four Figures are the results of DR framework with fixed $\mu$ when $\lambda \in[0.001,0.01,0.1,1,10,1000]$ and the bottom four Figures are the results of DR framework with fixed $\lambda$ when $\mu \in[0.001: 0.001: 0.009,0.01: 0.01: 0.1]$.

two trade-off parameters. To investigate this point, we conduct additional experiments by fixing the values of one parameter while changing the other one. More specifically, we choose four experiments including Exp.1, Exp.2, Exp.7, and Exp.8 as representatives and in these four experiments we fix one parameter and change the other parameter of DRFS-S, DRFST, and DRLS to conduct the experiments to report the WAR (recognition rate). The changing interval of $\lambda$ and $\mu$ are set as follows: $\lambda \in[0.001,0.01,0.1,1,10,1000]$ and $\mu \in[0.001$ : $0.001: 0.009,0.01: 0.01: 0.1]$. The fixed $\lambda$ and $\mu$ in the experiments are set as the values used in the experiments in Section IV-B. The experimental results are shown in Fig. 7. From Fig. 7, it is clear to see that the performance varies less with respect to the changes of $\lambda$ and $\mu$, respectively, for all four experiments, which indicates that our proposed DR framework is less sensitive to its trade-off parameters.

\section{E. Experiments with Deep Features}

Recently, the deep features extracted by trained deep neural networks, e.g., VGG [50] and ResNet [51], have achieved great success in many vision tasks. In this section, we investigate whether the deep features would benefit the performance of cross-database micro-expression recognition methods. To this end, we choose Exp.1 as the representative and use the recently released three-dimensional convolutional neural networks (3DCNNs) including C3D [52] and P3D ResNet [53], which are trained based on Sports 1-M dataset [54], to extract the feature corresponding to the last fully connected layer from microexpression samples instead of LBP-TOP to conduct additional experiments.

We show the experimental results of deep features and LBPTOP in Table VII. On one hand, it can from the results be seen that by using C3D and P3D ResNet features, the proposed
TABLE VII

EXPERIMENTAL RESULTS IN TERMS OF WAR/UAR OF DEEP FEATURES FOR CROSS-DATABASE MICRO-EXPRESSION RECOGNITION EXPERIMENTS, WHERE THE SOURCE DATASET IS CASME2 AND TARGET DATASET IS SMIC (HS).

\begin{tabular}{|c|c|c|c|}
\hline Feature & LBP-TOP [9] & C3D [52] & P3D ResNet [53] \\
\hline \hline SVM & $42.07 / 37.91$ & $32.93 / 27.31$ & $40.24 / 39.20$ \\
\hline \hline DRFS-S & $50.61 / 47.48$ & $48.17 / 43.09$ & $46.34 / 41.57$ \\
\hline DRFS-T & $47.56 / 42.37$ & $43.90 / 42.62$ & $46.34 / 47.28$ \\
\hline DRLS & $54.88 / 54.65$ & $44.51 / 41.88$ & $50.00 / 45.73$ \\
\hline Average & $51.02 / 48.17$ & $45.53 / 42.53$ & $47.56 / 44.86$ \\
\hline
\end{tabular}

three domain regenerators would achieve promisingly increase compared with the baseline method (SVM without any domain adaptation), which can demonstrate the effectiveness of the proposed DR framework. One the other hand, as the Table shows it is clear that the deep features do not outperform LBPTOP. However, we can find that in the case of using DRFST, P3D ResNet feature is very competitive against LBP-TOP and even better than LBP-TOP in term of UAR $(47.28 \%$ v.s. 42.37\%). It should be pointed out that C3D and P3D ResNet are trained on Sports 1-M, which is an action database and is very different from micro-expressions. This finding inspires us that it is very worthwhile to investigate the trained deep neural networks for micro-expression feature extraction, which may boost the performance of cross-database micro-expression recognition methods.

\section{Conclusion And Discussion}

In this paper, we have proposed a Domain Regeneration (DR) framework to deal with unsupervised cross-database micro-expression recognition problem, which is more challenging than conventional micro-expression recognition. By 
inputting source and target micro-expression samples, DR framework is able to regenerate the source and target samples such that the regenerated source and target microexpression samples would share the same or similar feature distributions. Consequently, the classifier learned based on the labeled source samples can accurately predict the microexpression categories of the target samples. Extensive crossdatabase micro-expression recognition experiments on SMIC and CASME II databases are conducted to evaluate the performance of the proposed DR framework. Experiments show that our DR framework can achieve overall satisfactory results and promisingly outperform recent proposed state-of-the-art crossdatabase emotion recognition methods. Additionally, based on our experiments, it is convincing that the class imbalance problem exists in target database and the heterogeneous problem between source and target databases are two important factors which affect the difficulty degree of cross-database microexpression recognition tasks.

With the development of deep learning techniques, a large number of deep transfer learning methods have been proposed and shown their promising performance in domain adaptation tasks in recent years [55], [56], [57]. One main reason of their success is that deep learning models have strong nonlinear mapping (representation) ability [58], which benefits bridging the source and target domains. In fact, by resorting to this ability, we are able to develop the deep learning version of domain regenerator under the DR framework, to deal with cross-database micro-expression recognition problem and in the future we will focus on investigating this point.

Finally, we would like to point out some limitations of our work. (1) In this paper, we focus on the domain regeneration models for solving cross-database micro-expression recognition problem. Actually, we are also able to investigate this challenging problem from the angle of microexpression features because features play an important role in the field of micro-expressin analysis. It would be a good way to develop effective micro-expression features including conventional handcraft features and emerging deep features for cross-database micro-expression recognition task. (2) In our experiments, due to the limitation of micro-expression databases, we just conduct three-category cross-database experiments. In fact, in order to deeply investigate cross-database micro-expression problem, more detailed categories based experiments (e.g., five micro-expressions) should be investigated when more micro-expression databases including more emotion categories are developed in the future.

\section{APPENDIX A}

TRADE-OFF PARAMETER SETTING FOR ULSIF, STM, AND DR FRAMEWORK IN THE EXPERIMENTS

Firstly, to make the readers understand the impact of the trade-off parameters of ULSIF and STM, we directly take their optimization problems from Eq. (23) in [41] and Eq. (1) in [24] and briefly introduce them in what follows.

uLSIF:

$$
\min _{\beta \in \mathbb{R}^{b}}\left[\frac{1}{2} \beta^{T} \hat{H} \beta-\hat{h}^{T} \beta+\frac{\lambda}{2} \beta^{T} \beta\right],
$$

where $\beta$ is the importance parameter and each of its elements corresponds to a source sample. The parameter $\lambda$ offers a trade-off between the original objective function of uLSIF and the $L 2$ norm based regularizer.

STM:

$$
\min _{\mathbf{w}, \mathbf{s}} R_{\mathbf{w}}\left(\mathcal{D}^{t r}, \mathbf{s}\right)+\lambda \Omega_{\mathbf{s}}\left(\mathbf{X}^{t r}, \mathbf{X}^{t e}\right),
$$

where $R_{\mathbf{w}}\left(\mathcal{D}^{t r}, \mathbf{s}\right)$ is the SVM empirical risk defined on source set $\mathcal{D}^{t r}$ with each sample weighted by $\mathbf{s} \in \mathbb{R}^{n_{t r}} . \Omega_{\mathbf{s}}\left(\mathbf{X}^{t r}, \mathbf{X}^{t e}\right)$ measures the feature distribution difference between source and target samples. The trade-off parameter $\lambda$ control the balance between the risk and the feature distribution difference terms.

In the experiments, we search the trade-off parameter $\lambda$ from from a preset parameter interval [1:1:100] $\times t(t=1$, $10,100,1000,10000,100000)$ for ULSIF and [0.01:0.01:0.09, $0.1: 0.1: 0.9,1: 1: 15]$ for STM and report their results of optimal trade-off parameter corresponding to highest WAR. Herein we give the corresponding optimal trade-off parameters used in our experiments.

For uLSIF, the optimal $\lambda$ are $570,36 \times 10^{5}, 89,1,79$, and $42 \times 10^{5}$ for TYPE-I (Exp.1, $\cdots$, Exp.6) and $7 \times 10^{4}, 4 \times 10^{3}$, $53 \times 10^{4}, 1,17 \times 10^{4}$, and $17 \times 10^{5}$ for TYPE-II $(\operatorname{Exp} .7, \cdots$, Exp.12).

For STM, the obtained trade-off parameter $\lambda$ are 4, 0.04, 8, 0.02, 15, and 0.03 for TYPE-I (Exp.1, ․ Exp.6) and 2, 0.8, $0.5,11,1$, and 0.1 for TYPE-II (Exp.7, $\cdots$, Exp.12).

Finally, for three DR framework based methods, we search $\lambda$ from $[0.001,0.01,0.1,1,10,100,1000]$ and $\mu$ from [0.001:0.001:0.009, 0.01:0.01:0.09, 0.1:0.1:1, 2:10]. we give the optimal trade-off parameters $(\lambda, \mu)$ for the proposed DR framework (DRFS-S, DRFS-T, and DRLS) used in the experiments.

For DRFS-S, the optimal parameters $(\lambda, \mu)$ are $(0.001$, $0.03),(0.1,0.007),(1,0.07),(0.01,0.005),(0.01,0.003)$, and $(10,0.001)$ for TYPE-I (Exp.1, $\cdots$, Exp.6) and (1, 0.03), (100, $0.03),(1,0.5),(1,0.08),(0.01,0.06)$, and $(100,0.2)$ for TYPEII (Exp.7, $\cdots$, Exp.12).

For DRFS-T, the optimal parameters $(\lambda, \mu)$ are $(100,0.009)$, $(1,0.003),(10,0.001),(100,0.2),(0.01,0.001)$, and $(1,0.005)$ for TYPE-I (Exp.1, $\cdots$, Exp.6) and (10, 0.06), (10, 0.3), (1, $0.2),(10,0.02),(10,0.04)$, and $(1,0.5)$ for TYPE-II (Exp.7, $\cdots$, Exp.12).

For DRLS, the optimal parameters $(\lambda, \mu)$ are $(1,0.1),(10$, $3),(0.1,0.5),(0.1,0.6),(10,0.06)$, and $(1,0.001)$ for TYPE-I (Exp.1, ... Exp.6) and $(0.1,0.1),(10,0.1),(10,5),(1,0.3)$, $(100,4)$, and $(1000,2)$ for TYPE-II (Exp.7, $\cdots$, Exp.12).

\section{ACKNOWLEDGMENT}

The authors would like to thank Mr. Chuangao Tang and Mr. Tengfei Song from Southeast University, China and Mr. Henglin Shi from University of Oulu, Finland for helping extract deep features used in the experiments. The authors also gratefully acknowledge the support of NVIDIA Corporation with the donation of the Titan X Pascal GPU used for this research. 


\section{REFERENCES}

[1] T. Pfister, X. Li, G. Zhao, and M. Pietikäinen, "Recognising spontaneous facial micro-expressions," in International Conference on Computer Vision. IEEE, 2011, pp. 1449-1456.

[2] M. Frank, M. Herbasz, K. Sinuk, A. Keller, and C. Nolan, "I see how you feel: Training laypeople and professionals to recognize fleeting emotions," in The Annual Meeting of the International Communication Association. Sheraton New York, New York City, 2009.

[3] M. G. Frank, C. J. Maccario, and V. Govindaraju, "Behavior and security," Protecting Airline Passengers in the Age of Terrorism. Greenwood Pub Group, Santa Barbara, California, pp. 86-106, 2009.

[4] M. OSullivan, M. G. Frank, C. M. Hurley, and J. Tiwana, "Police lie detection accuracy: The effect of lie scenario," Law and Human Behavior, vol. 33, no. 6, pp. 530-538, 2009.

[5] X. Li, X. Hong, A. Moilanen, X. Huang, T. Pfister, G. Zhao, and M. Pietikäinen, "Towards reading hidden emotions: A comparative study of spontaneous micro-expression spotting and recognition methods," IEEE Transactions on Affective Computing, 2017.

[6] P. Lu, W. Zheng, Z. Wang, Q. Li, Y. Zong, M. Xin, and L. Wu, "Microexpression recognition by regression model and group sparse spatiotemporal feature learning," IEICE TRANSACTIONS on Information and Systems, vol. 99, no. 6, pp. 1694-1697, 2016.

[7] K. Yan, W. Zheng, Z. Cui, and Y. Zong, "Cross-database facial expression recognition via unsupervised domain adaptive dictionary learning," in International Conference on Neural Information Processing. Springer, 2016, pp. 427-434

[8] Y. Zong, X. Huang, W. Zheng, Z. Cui, and G. Zhao, "Learning a target sample re-generator for cross-database micro-expression recognition," in Proceedings of the 2017 ACM on Multimedia Conference. ACM, 2017, pp. 872-880.

[9] G. Zhao and M. Pietikäinen, "Dynamic texture recognition using local binary patterns with an application to facial expressions," IEEE Transactions on Pattern Analysis and Machine Intelligence, vol. 29, no. 6, pp. 915-928, 2007.

[10] J. A. Ruiz-Hernandez and M. Pietikäinen, "Encoding local binary patterns using the re-parametrization of the second order gaussian jet," in Proceedings of the 10th IEEE International Conference and Workshops on Automatic Face and Gesture Recognition $(F G)$. IEEE, 2013, pp. $1-6$.

[11] Y. Wang, J. See, R. C.-W. Phan, and Y.-H. Oh, "Lbp with six intersection points: Reducing redundant information in lbp-top for micro-expression recognition," in Asian Conference on Computer Vision. Springer, 2014, pp. 525-537.

[12] X. Huang, S.-J. Wang, G. Zhao, and M. Pietikäinen, "Facial microexpression recognition using spatiotemporal local binary pattern with integral projection," in Proceedings of the IEEE International Conference on Computer Vision Workshops, 2015, pp. 1-9.

[13] X. Huang, G. Zhao, X. Hong, W. Zheng, and M. Pietikäinen, "Spontaneous facial micro-expression analysis using spatiotemporal completed local quantized patterns," Neurocomputing, vol. 175, pp. 564-578, 2016.

[14] Y.-J. Liu, J.-K. Zhang, W.-J. Yan, S.-J. Wang, G. Zhao, and X. Fu, "A main directional mean optical flow feature for spontaneous microexpression recognition," IEEE Transactions on Affective Computing, vol. 7, no. 4, pp. 299-310, 2016.

[15] F. Xu, J. Zhang, and J. Z. Wang, "Microexpression identification and categorization using a facial dynamics map," IEEE Transactions on Affective Computing, vol. 8, no. 2, pp. 254-267, 2017.

[16] S.-J. Wang, W.-J. Yan, G. Zhao, X. Fu, and C.-G. Zhou, "Microexpression recognition using robust principal component analysis and local spatiotemporal directional features," in Workshop at the European Conference on Computer Vision. Springer, 2014, pp. 325-338.

[17] J. Wright, A. Ganesh, S. Rao, Y. Peng, and Y. Ma, "Robust principal component analysis: Exact recovery of corrupted low-rank matrices via convex optimization," in Advances in Neural Information Processing Systems, 2009, pp. 2080-2088.

[18] S.-J. Wang, W.-J. Yan, X. Li, G. Zhao, and X. Fu, "Micro-expression recognition using dynamic textures on tensor independent color space," in 22nd International Conference on Pattern Recognition (ICPR). IEEE, 2014, pp. 4678-4683.

[19] S.-J. Wang, W.-J. Yan, X. Li, G. Zhao, C.-G. Zhou, X. Fu, M. Yang, and J. Tao, "Micro-expression recognition using color spaces," IEEE Transactions on Image Processing, vol. 24, no. 12, pp. 6034-6047, 2015.

[20] P. Ekman and W. V. Friesen, "Facial action coding system," 1977.

[21] D. H. Kim, W. J. Baddar, and Y. M. Ro, "Micro-expression recognition with expression-state constrained spatio-temporal feature representa- tions," in Proceedings of the 2016 ACM on Multimedia Conference. ACM, 2016, pp. 382-386.

[22] A. Krizhevsky, I. Sutskever, and G. E. Hinton, "Imagenet classification with deep convolutional neural networks," in Advances in Neural Information Processing Systems, 2012, pp. 1097-1105.

[23] F. A. Gers, N. N. Schraudolph, and J. Schmidhuber, "Learning precise timing with 1stm recurrent networks," Journal of Machine Learning Research, vol. 3, no. Aug, pp. 115-143, 2002.

[24] W.-S. Chu, F. De la Torre, and J. F. Cohn, "Selective transfer machine for personalized facial action unit detection," in Proceedings of the IEEE Conference on Computer Vision and Pattern Recognition, 2013, pp. $3515-3522$

[25] — "Selective transfer machine for personalized facial expression analysis," IEEE Transactions on Pattern Analysis and Machine Intelligence, vol. 39, no. 3, pp. 529-545, 2017.

[26] E. Sangineto, G. Zen, E. Ricci, and N. Sebe, "We are not all equal: Personalizing models for facial expression analysis with transductive parameter transfer," in Proceedings of the 22nd ACM International Conference on Multimedia. ACM, 2014, pp. 357-366.

[27] W. Zheng and X. Zhou, "Cross-pose color facial expression recognition using transductive transfer linear discriminat analysis," in IEEE International Conference on Image Processing (ICIP). IEEE, 2015, pp. 1935-1939.

[28] W. Zheng, Y. Zong, X. Zhou, and M. Xin, "Cross-domain color facial expression recognition using transductive transfer subspace learning," IEEE Transactions on Affective Computing, 2016.

[29] B. Schuller, B. Vlasenko, F. Eyben, M. Wollmer, A. Stuhlsatz, A. Wendemuth, and G. Rigoll, "Cross-corpus acoustic emotion recognition: Variances and strategies," IEEE Transactions on Affective Computing, vol. 1, no. 2, pp. 119-131, 2010.

[30] A. Hassan, R. Damper, and M. Niranjan, "On acoustic emotion recognition: compensating for covariate shift," IEEE Transactions on Audio, Speech, and Language Processing, vol. 21, no. 7, pp. 1458-1468, 2013.

[31] J. Deng, Z. Zhang, E. Marchi, and B. Schuller, "Sparse autoencoderbased feature transfer learning for speech emotion recognition," in 2013 Humaine Association Conference on Affective Computing and Intelligent Interaction (ACII). IEEE, 2013, pp. 511-516.

[32] J. Deng, Z. Zhang, F. Eyben, and B. Schuller, "Autoencoder-based unsupervised domain adaptation for speech emotion recognition," IEEE Signal Processing Letters, vol. 21, no. 9, pp. 1068-1072, 2014.

[33] J. Deng, X. Xu, Z. Zhang, S. Frühholz, and B. Schuller, "Universum autoencoder-based domain adaptation for speech emotion recognition," IEEE Signal Processing Letters, vol. 24, no. 4, pp. 500-504, 2017.

[34] Z. Huang, W. Xue, Q. Mao, and Y. Zhan, "Unsupervised domain adaptation for speech emotion recognition using pcanet," Multimedia Tools and Applications, pp. 1-15, 2016.

[35] P. Song, W. Zheng, S. Ou, X. Zhang, Y. Jin, J. Liu, and Y. Yu, "Crosscorpus speech emotion recognition based on transfer non-negative matrix factorization," Speech Communication, vol. 83, pp. 34-41, 2016.

[36] Y. Zong, W. Zheng, T. Zhang, and X. Huang, "Cross-corpus speech emotion recognition based on domain-adaptive least-squares regression," IEEE Signal Processing Letters, vol. 23, no. 5, pp. 585-589, 2016.

[37] H. Sagha, J. Deng, M. Gavryukova, J. Han, and B. Schuller, "Cross lingual speech emotion recognition using canonical correlation analysis on principal component subspace," in IEEE International Conference on Acoustics, Speech and Signal Processing (ICASSP). IEEE, 2016, pp. 5800-5804.

[38] S. J. Pan and Q. Yang, "A survey on transfer learning," IEEE Transactions on Knowledge and Data Engineering, vol. 22, no. 10, pp. 13451359, 2010.

[39] J. Huang, A. Gretton, K. M. Borgwardt, B. Schölkopf, and A. J. Smola, "Correcting sample selection bias by unlabeled data," in Advances in Neural Information Processing Systems, 2006, pp. 601-608.

[40] M. Sugiyama, S. Nakajima, H. Kashima, P. V. Buenau, and M. Kawanabe, "Direct importance estimation with model selection and its application to covariate shift adaptation," in Advances in Neural Information Processing Systems, 2008, pp. 1433-1440.

[41] T. Kanamori, S. Hido, and M. Sugiyama, "A least-squares approach to direct importance estimation," The Journal of Machine Learning Research, vol. 10, pp. 1391-1445, 2009.

[42] K. M. Borgwardt, A. Gretton, M. J. Rasch, H.-P. Kriegel, B. Schölkopf, and A. J. Smola, "Integrating structured biological data by kernel maximum mean discrepancy," Bioinformatics, vol. 22, no. 14, pp. e49e57, 2006.

[43] G. Casella and R. L. Berger, Statistical inference. Duxbury Pacific Grove, CA, 2002, vol. 2. 
[44] R. O. Duda, P. E. Hart, and D. G. Stork, Pattern classification. John Wiley \& Sons, 2012.

[45] S. Ji and J. Ye, "An accelerated gradient method for trace norm minimization," in Proceedings of the 26th annual International Conference on Machine Learning. ACM, 2009, pp. 457-464.

[46] Z. Lin, M. Chen, and Y. Ma, "The augmented lagrange multiplier method for exact recovery of corrupted low-rank matrices," arXiv preprint arXiv: $1009.5055,2010$.

[47] X. Li, T. Pfister, X. Huang, G. Zhao, and M. Pietikäinen, "A spontaneous micro-expression database: Inducement, collection and baseline," in Proceedings of the 10th IEEE International Conference and Workshops on Automatic Face and Gesture Recognition (FG). IEEE, 2013, pp. 1-6, http://www.cse.oulu.fi/SMICDatabase.

[48] W.-J. Yan, X. Li, S.-J. Wang, G. Zhao, Y.-J. Liu, Y.-H. Chen, and X. Fu, "Casme II: An improved spontaneous micro-expression database and the baseline evaluation," PloS one, vol. 9, no. 1, p. e86041, 2014, http: //fu.psych.ac.cn/CASME/casme2-en.php.

[49] G. Zhao and M. Pietikäinen, "Boosted multi-resolution spatiotemporal descriptors for facial expression recognition," Pattern Recognition Letters, vol. 30, no. 12, pp. 1117-1127, 2009.

[50] K. Simonyan and A. Zisserman, "Very deep convolutional networks for large-scale image recognition," arXiv preprint arXiv:1409.1556, 2014.

[51] K. He, X. Zhang, S. Ren, and J. Sun, "Deep residual learning for image recognition," in Proceedings of the IEEE conference on computer vision and pattern recognition, 2016, pp. 770-778.

[52] D. Tran, L. Bourdev, R. Fergus, L. Torresani, and M. Paluri, "Learning spatiotemporal features with $3 \mathrm{~d}$ convolutional networks," in Proceedings of the IEEE international conference on computer vision, 2015, pp $4489-4497$.

[53] Z. Qiu, T. Yao, and T. Mei, "Learning spatio-temporal representation with pseudo-3d residual networks," in Proceedings of the International Conference on Computer Vision (ICCV), vol. 8, 2017.

[54] A. Karpathy, G. Toderici, S. Shetty, T. Leung, R. Sukthankar, and L. Fei-Fei, "Large-scale video classification with convolutional neural networks," in Proceedings of the IEEE conference on Computer Vision and Pattern Recognition, 2014, pp. 1725-1732.

[55] X. Shu, G.-J. Qi, J. Tang, and J. Wang, "Weakly-shared deep transfer networks for heterogeneous-domain knowledge propagation," in Proceedings of the 23rd ACM international conference on Multimedia. ACM, 2015, pp. 35-44.

[56] Z. Wang, J. Yang, H. Jin, E. Shechtman, A. Agarwala, J. Brandt, and T. S. Huang, "Deepfont: Identify your font from an image," in Proceedings of the 23rd ACM international conference on Multimedia. ACM, 2015, pp. 451-459.

[57] M. Long, J. Wang, Y. Cao, J. Sun, and S. Y. Philip, "Deep learning of transferable representation for scalable domain adaptation," IEEE Transactions on Knowledge and Data Engineering, vol. 28, no. 8, pp. 2027-2040, 2016

[58] Y. LeCun, Y. Bengio, and G. Hinton, "Deep learning," Nature, vol. 521, no. 7553, pp. 436-444, 2015

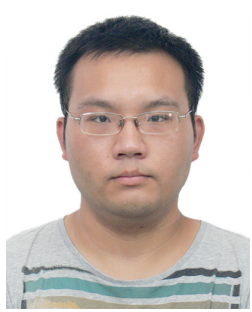

Yuan Zong received the BS and MS degrees in electronics engineering both from Nanjing Normal University, Nanjing, Jiangsu, China, in 2011 and 2014 , respectively. He is currently working toward the $\mathrm{PhD}$ degree in the Key Laboratory of Child Development and Learning Science of Ministry of Education, School of Biological Sciences and Medical Engineering, Southeast University, Nanjing, Jiangsu, China. From October 2016 to September 2017, he was working as a visiting student at the Center for Machine Vision and Signal Analysis, University of Oulu, Finland. His research interests include affective computing, pattern recognition, and computer vision.

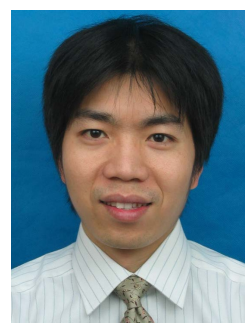

Wenming Zheng received the BS degree in computer science from Fuzhou University, Fujian, China, in 1997, the MS degree in computer science from Huaqiao University, Quanzhou, Fujian, China in 2001, and the PhD degree in signal processing from Southeast University, Nanjing, Jiangsu, China, in 2004. Since 2004, he has been with the Research Center for Learning Science, Southeast University. $\mathrm{He}$ is currently a professor in the Key Laboratory of Child Development and Learning Science of the Ministry of Education, School of Biological Sciences and Medical Engineering, Southeast University. His research interests include neural computation, pattern recognition, machine learning, and computer vision. He is a member of the IEEE.

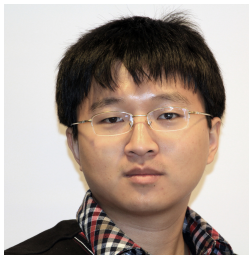

Xiaohua Huang received the BS degree in communication engineering from Huaqiao University, Quanzhou, Fujian, China in 2006. He received his $\mathrm{PhD}$ degree in computer science and engineering from University of Oulu, Oulu, Finland in 2014. He was a research assistant in Southeast University since 2006. He has been a scientist researcher in the Center for Machine Vision and Signal Analysis at University of Oulu, Finland since 2015. He has authored or co-authored more than 20 papers in journals and conferences, and has served as a reviewer for journals and conferences. His current research interests include facial expression recognition, micro-expression analysis, group-level emotion recognition, multi-modal emotion recognition and texture classification.

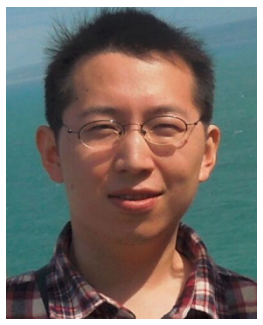

Jingang Shi received the $\mathrm{BS}$ degree and $\mathrm{PhD}$ degree both from the School of Electronic and Information Engineering, Xi'an Jiaotong University, Xi' an, Shaanxi, China. Since 2017, he has been a postdoctoral researcher at the Center for Machine Vision Research and Signal Analysis, University of Oulu, Finland. His current research interests mainly include image restoration and face analysis.

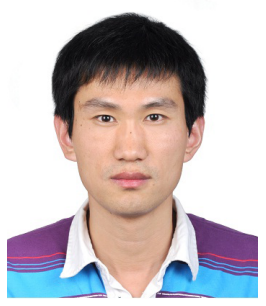

Zhen Cui received the BS, MS, and $\mathrm{PhD}$ degrees from Shandong Normal University, Sun Yat-sen University, and Institute of Computing Technology (ICT), Chinese Academy of Sciences in 2004, 2006 , and 2014, respectively. He was a Research Fellow in the Department of Electrical and Computer Engineering at National University of Singapore (NUS) from 2014 to 2015. Currently, he is a professor of Nanjing University of Science and Technology, Nanjing, Jiangsu, China. His research interests mainly include Sparse Coding, Manifold Learning, Deep Learning, Face Detection, Alignment \& Recognition, Image Super Resolution, etc. 


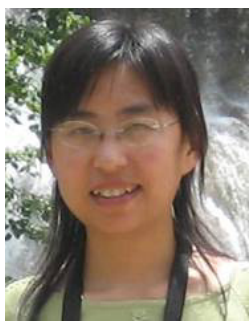

Guoying Zhao (SM'12) is currently a Professor with the Center for Machine Vision and Signal Analysis, University of Oulu, Finland, where she has been a senior researcher since 2005 and an Associate Professor since 2014. She received the Ph.D. degree in computer science from the Chinese Academy of Sciences, Beijing, China, in 2005. In 2011, she was selected to the highly competitive Academy Research Fellow position. She was Nokia visiting professor in 2016. She has authored or co-authored more than 160 papers in journals and conferences. Her papers have currently over 6900 citations in Google Scholar (h-index 35). She is co-publicity chair for FG2018, has served as area chairs for several conferences and is associate editor for Pattern Recognition, IEEE Transactions on Circuits and Systems for Video Technology, and Image and Vision Computing Journals. She has lectured tutorials at ICPR 2006, ICCV 2009, and SCIA 2013, authored/edited three books and seven special issues in journals. Dr. Zhao was a Co-Chair of many International Workshops at ECCV, ICCV, CVPR, ACCV and BMVC. Her current research interests include image and video descriptors, facial-expression and micro-expression recognition, gait analysis, dynamic-texture recognition, human motion analysis, and person identification. Her research has been reported by Finnish TV programs, newspapers and MIT Technology Review. 